\title{
Origen de la calidad del agua del acuífero colgado y su relación con los cambios de uso de suelo en el Valle de San Luis Potosí
}

\author{
Briseida López-Álvarez ${ }^{1,2, *}$, José Alfredo Ramos-Leal ${ }^{2}$, Janete Moran-Ramírez ${ }^{2}$, Antonio \\ Cardona Benavides ${ }^{3}$, Guillermo Hernández Garcia ${ }^{4}$ \\ ${ }^{1}$ Programa Agua y Sociedad. Colegio de San Luis, A. C. (COLSAN). Parque de Macul No. 155, Fracc. Colinas del Parque, San Luis \\ Potosí, S.L.P. México, C.P. 78299. \\ ${ }^{2}$ Geociencias Aplicadas. Instituto Potosino de Investigación Científica y Tecnológica, A.C. (IPICYT), C. Presa San José \# 2055, \\ Lomas 4a. Sección, CP 78216, San Luís Potosí, SLP, México. \\ ${ }^{3}$ Área de Ciencias de la Tierra de la Facultad de ingeniería, Universidad Autónoma de San Luis Potosí. \\ ${ }^{4}$ Instituto de Geofísica, Universidad Nacional Autónoma de México. Ciudad Universitaria, 04510 Coyoacán, México, D. F. \\ *blopez@colsan.edu.mx
}

\section{Resumen}

La historia de la ciudad San Luis Potosí se remonta al siglo XVI. Con el descubrimiento de yacimientos de oro y plata y la presencia de cuerpos de agua en el valle, fue fundada la ciudad San Luis Minas del Potosí, dando lugar a los dos primeros usos de suelo, urbano y minero. A partir del siglo XVII, el uso de suelo agrícola se desarrolló en huertos y fue relegado a la periferia de la zona urbana en el transcurso del tiempo. Finalmente el uso de suelo industrial surgió de manera importante en la segunda mitad del siglo XX. En la actualidad los tres usos de suelo existentes dentro del Valle de San Luis Potosí son el urbano, agrícola e industrial.

A través de una campaña de muestreo hidrogeoquímico en octubre de 2008, con 44 muestras de norias y 3 de manantiales dentro del valle, se evaluaron parámetros físico-químicos, cationes, aniones y elementos traza.

En los tres usos de suelo en la zona de estudio fueron detectados niveles importantes de nitratos, sulfatos, cloruros, conductividad eléctrica, coliformes totales y fecales; sin embargo, en la zona urbana existen anomalías puntuales de metales pesados principalmente de mercurio, bario, estroncio, cadmio, plomo, fósforo y plata, relacionadas a las antiguas actividades mineras y a la industria activa en la zona. Mientras que en la zona agrícola, la presencia de metales está asociada a los canales a cielo abierto que también reciben agua del Tanque Tenorio y éste a su vez de la zona industrial. En la zona industrial se detectaron grandes anomalías de tipo puntual en casi todos los metales pesados analizados; la principal fuente de estos contaminantes corresponden a un terreno industrial activo.

Este trabajo está enfocado a evaluar el impacto que ha generado la actividad antropogénica sobre el acuífero colgado del Valle de San Luis Potosí desde inicios de la fundación de la ciudad hasta la actualidad, utilizando la calidad del agua como herramienta de análisis.

Palabras clave: Cambio de uso de suelo, calidad del agua, acuífero colgado, actividad minera, contaminación.

\footnotetext{
Abstract

The history of San Luis Potosi City dates back to the sixteenth century. With the discovery of gold and silver deposits and the presence of water bodies in the valley, the city of San Luis Minas Potosi was founded, leading to the first two uses of land: urban and mining. From the seventeenth century, agricultural land developed in orchards and, over time, was relegated to the periphery of the urban area. Finally, industrial land use emerged significantly in the second half of the twentieth century. Currently the three existing land uses within the Valley of San Luis Potosi are urban, agricultural and industrial.

Through a hydrogeochemical sampling campaign in October 2008 with 44 samples from wells and 3 from springs within the valley, we assessed physical and chemical parameters, cations, anions and trace elements.
} 
In the three land uses within the study area, we detected significant levels of nitrates, sulphates, chlorides, electrical conductivity, total and fecal coliforms; but in urban areas there are punctual anomalies of heavy metals, mainly mercury, barium, strontium, cadmium, lead, phosphorus and silver related to former mining and active industry in the area. However, in the agricultural zone, the presence of metals is associated with open channels, which also receive water from the Tanque Tenorio and this in turn from the industrial area. In the industrial area, puntual anomalies were detected in almost all heavy analyzed metals; the main source of these pollutants corresponds to an active industrial area.

This work aims to evaluate the impact of anthropogenic activity in the perched aquifer of the Valley of San Luis Potosi since the city's foundation to the present, using water quality as an analytical tool.

Keywords: Change of land use, water quality, perched aquifer, mining, pollution.

\section{Introducción}

La composición química de agua subterránea puede ser controlada por factores naturales o antropogénicos. Los naturales son el clima, su interacción con el medio geológico por el cual circula y el tiempo de residencia. Los antropogénicos están directamente relacionados con fuentes potenciales de contaminación asociadas a actividades humanas.

Desde el siglo XVIII, pero más intensamente en los últimos 50 años, los seres humanos han transformado los ecosistemas del mundo más rápido y extensamente que en ningún otro periodo comparable de la historia. Ahora se reconoce que, aún cuando los cambios de uso de suelo ocurren a nivel local, pueden tener consecuencias globales en el ciclo hidrogeológico, debido a que se ha demostrado que la cantidad y calidad del agua dependen en gran parte del uso que se da al suelo en las áreas que captan, conducen, almacenan, proveen y renuevan el recurso hídrico (SEMARNAT, 2005). Sin embargo, como consecuencia del crecimiento poblacional y el desarrollo económico, se generan presiones que conducen a cambios en el uso del suelo de las cuencas (SEMARNAT, 2005).

Diversas investigaciones han sido realizadas en varias partes del mundo orientadas a establecer la relación entre los usos de suelo y sus afectaciones en el agua subterránea (Schmidt y Sherman, 1987; Dummer y Straaten, 1988; Charbonneau y Kondolf, 1993; Cox et al., 1996; Barber et al., 1996; Tong y Chen, 2002; Liu et al., 2005 y Naik et al., 2008).

Un ejemplo de las alteraciones a un sistema natural propiciadas por actividades antropogénicas es el Valle de San Luis Potosí (VSLP) (Figura 1), en donde los usos de suelo han constituido un factor de alto impacto en el volumen y calidad del sistema acuífero. Aunada a esta situación, los cambios de uso de suelo, desde los primeros asentamientos humanos en el valle, han propiciado un efecto de contaminación cruzada que vuelve más complejo el problema de contaminación en el agua subterránea.

La historia del Valle de San Luis Potosí (VSLP) se remonta al siglo XVI, en la región conocida como la Gran Chichimeca, que era diversa tanto en grupos étnicos nómadas como en su clima, flora y fauna, geografía y relieve (Galván-Arellano, 1999). El agua estaba concentrada en algunos puntos y de las serranías bajaban arroyos naturales que temporalmente llevaban agua (Galván-Arellano, 1999). Una muestra de este escenario era la subregión conocida como el Gran Tunal (hoy VSLP). Con el proceso de pacificación y colonización de los nativos, el descubrimiento de recursos mineros (yacimientos de oro y plata) en el llamado Cerro de San Pedro Potosí y la presencia de cuerpos de agua en el valle, fue fundada la ciudad, entonces nombrada, San Luis Minas del Potosí (actual ciudad de San Luis Potosí), el 3 de Noviembre de 1592. La minería fue el principal detonador del crecimiento y desarrollo de la nueva población, y además la causa de grandes modificaciones del entorno. Por ejemplo, una gran deforestación de las serranías próximas debido a la explotación de leña como combustible para atender la demanda de la minería y de la población. La agricultura fue la segunda actividad económica que más adelante representaría un factor de impacto importante en la calidad del agua, no sólo superficial sino subterránea, dicha actividad se desarrollaba dentro de la zona urbana en huertos a partir del siglo XVII (Figura 2). La tercera actividad económica fue la industrial, que surgió de manera importante en la segunda mitad del siglo XX.

Con la urbanización se generaron alteraciones al sistema hidrológico, una muestra de ello fueron las grandes inundaciones que se registraron desde los primeros años de la fundación de la ciudad de San Luís las Minas Potosí, lo que propició la construcción de una obra de desvío llamada "la corriente", en 1688. Ésta desalojaba a las aguas que bajaban de la Sierra de San Miguelito (SSM) e inundaban a la ciudad. Una segunda obra de desvío fue realizada en 1771. Ambas obras funcionaron hasta 1946 (De la RosaCharcas y Llanas-Rivera, 2000).

Desde sus inicios, la ciudad de San Luis Potosí (SLP) se abastecía de fuentes de agua superficiales: pequeños manantiales, ríos, arroyos, incluso de charcos que se formaban en temporadas de lluvia, teniendo un ecosistema aún en equilibrio. Al agotarse las fuentes de abastecimiento inmediatas en el valle, fueron necesarias obras hidráulicas para dar abasto a la ciudad en constante crecimiento, como el acueducto, las "conservas" (cajas de agua) y la presa 


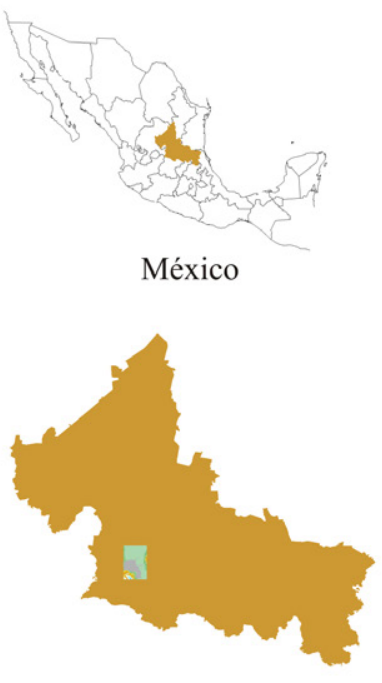

San Luis Potosí

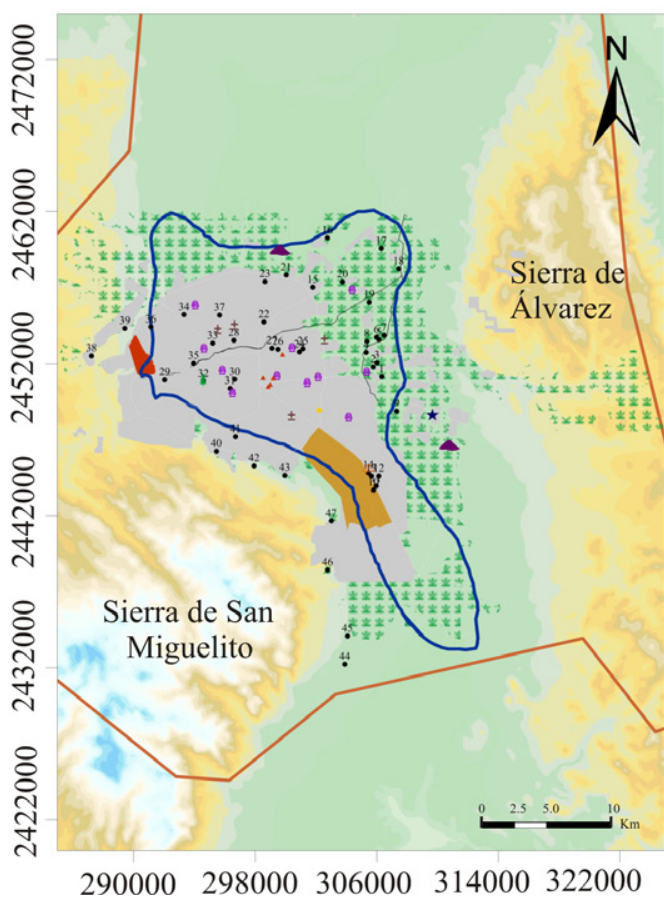

Símbología

Relieve topográfico

Vías de comunicación

Uso de suelo urbano en 2005

Uso de suelo agrícola

Uso de suelo industrial

Industria minera

Sitio de muestra

$\star \quad$ Tanque tenorio

- Jales de fluorita

- Basureros municipales

㗊 Gasolineras

$\Delta$ Antiguos residuos mineros

\pm Cementerios

$\triangle$ Terreno Industrial Activo

奉 Parque Morales

_ - Río Santiago (pavimentado)

- Canal

— Acuífero somero

_ Cuenca administrativa

Figura 1. Localización del área de estudio, fuentes potenciales de contaminación y usos de suelo,

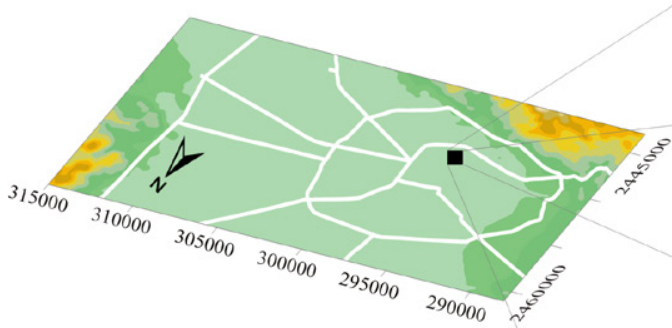

Simbología

Uso de suelo urbano

Uso de suelo agrícola

Uso de suelo minero

Cuerpos de agua

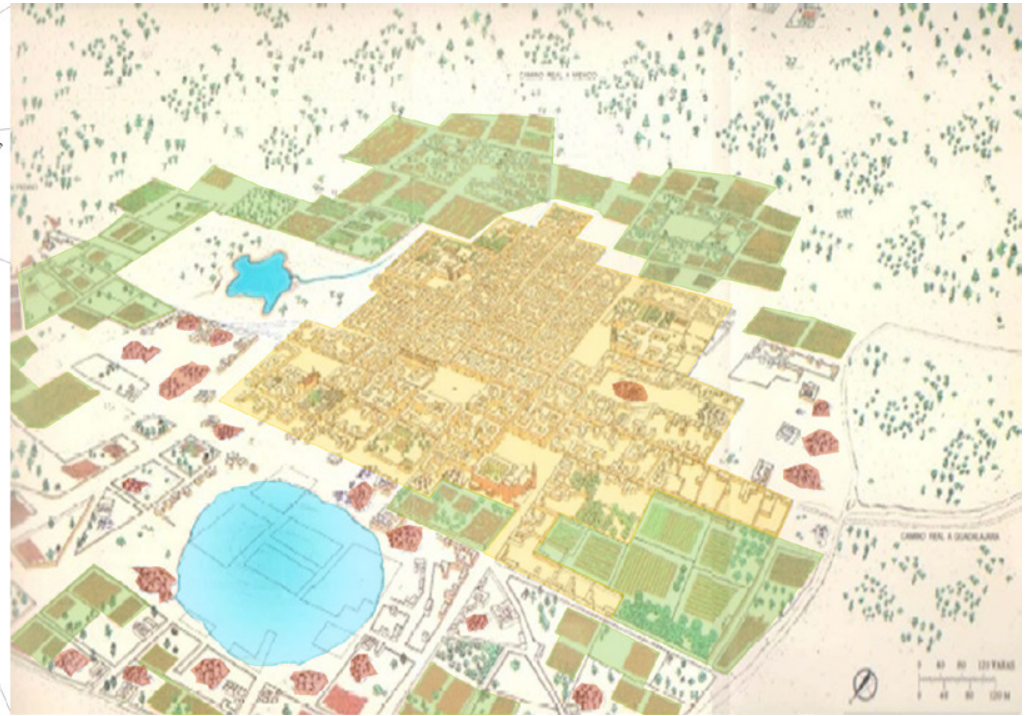

Figura 2. Primeros usos de suelo en la ciudad de San Luis Potosí en el siglo VXII.
San José.

El acueducto fue construido en 1831 llevando agua de la SSM a "las conservas", obras construidas en 1835 destinadas a almacenar agua, sirviendo para el abastecimiento público y funcionaron hasta la primera mitad del siglo XX. La presa San José comenzó a funcionar en 1903 y en la actualidad es la principal fuente de abasto de agua superficial para la zona conurbada del VSLP, aprovechando las afluentes del Río Santiago.

En el siglo XIX, se dio inicio al uso activo de agua subterránea como fuente de abastecimiento. Desde inicios de éste siglo las norias daban servicio a la población para uso doméstico e irrigación de pequeños huertos (SheridanPrieto, 2001).

Hasta mediados del siglo pasado los cambios sufridos en el VSLP habían sido relativamente paulatinos. Sin embargo, a cuatro siglos de la fundación de la ciudad de SLP se han experimentado cambios muy drásticos en los usos de suelo que a su vez han tenido un gran impacto en el sistema hidrológico en los últimos 50 años.

La zona conurbada del VSLP formada por los municipios de San Luis Potosí y Soledad de Graciano Sánchez (SGS), 
incrementó 15 veces su tamaño de 1959 a 2005 (Figura 3), registrándose el cambio de superficie urbana más importante entre 1970 y 1993 al cuadruplicarse (Noyola et al., 2009). En cuanto a la población de estos dos municipios, para el año 2000 equivalían al $38 \%$ de la población total de estado. Con base en el último censo poblacional (INEGI, 2010), representan $40 \%$ de la población total estatal.

Este trabajo está enfocado a evaluar el impacto que ha generado la actividad antropogénica sobre el acuífero colgado del VSLP desde inicios de la fundación de la ciudad hasta la actualidad, utilizando la calidad del agua como herramienta de análisis.

\section{Descripción del área de estudio}

\subsection{Localización}

El VSLP se encuentra limitado orográficamente al Norte por un conjunto de cerros llamados "Alto La Melada", al Occidente y Sur por la SSM y al Oriente por la Sierra de Álvarez (Figura 1).

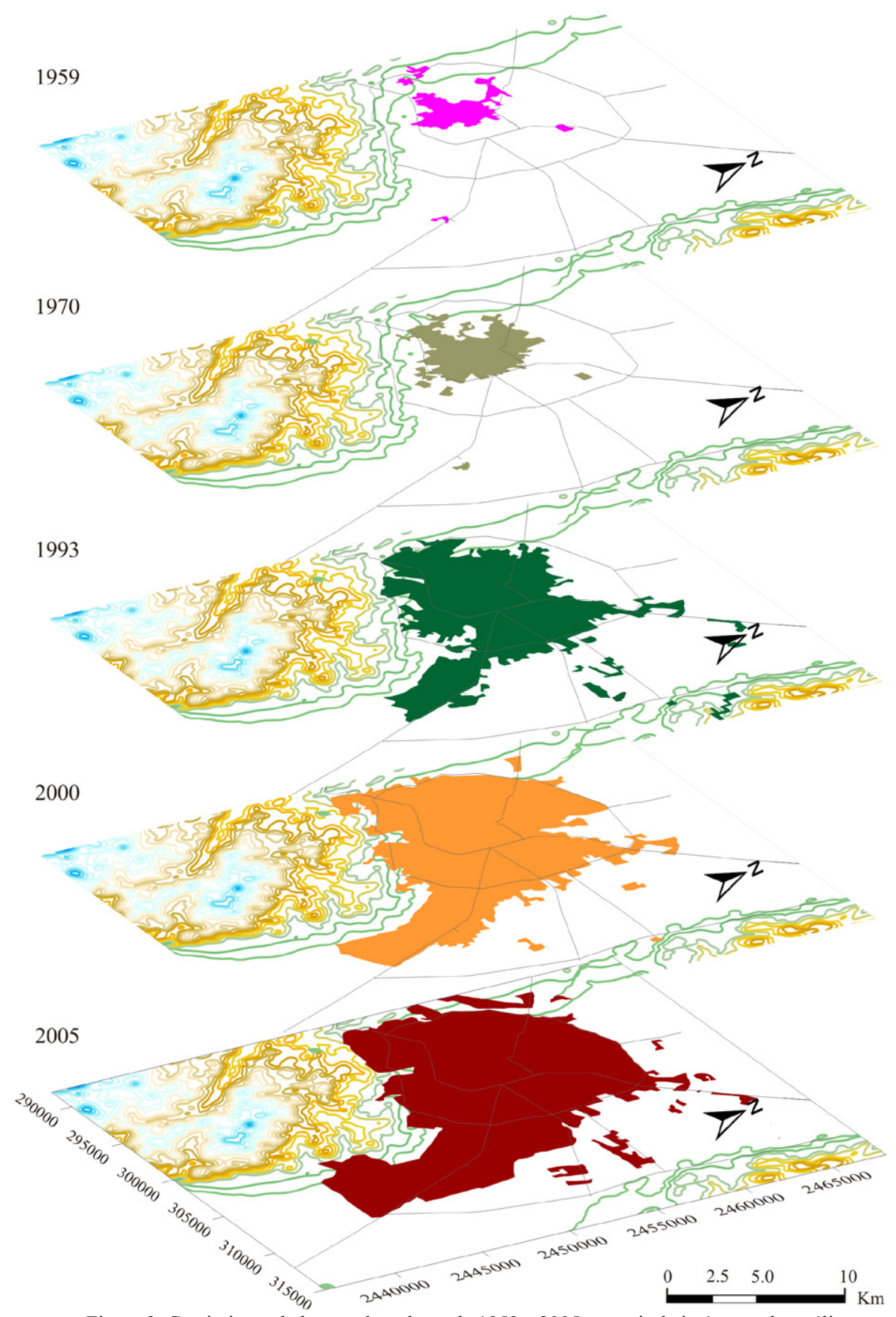

Figura 3. Crecimiento de la mancha urbana de 1959 a 2005, a partir de imágenes de satélite. 
El sistema acuífero del VSLP forma parte de la cuenca hidrológica No. 37 denominada "El Salado" con coordenadas geográficas $21^{\circ} 54^{\prime} 54^{\prime \prime}$ a $22^{\circ} 28^{\prime} 15^{\prime \prime}$ de latitud Norte y $101^{\circ} 13^{\prime} 44^{\prime \prime}$ a $100^{\circ} 37^{\prime} 08^{\prime \prime}$, en la porción SurOccidental del estado que lleva el mismo nombre.

Inmersa en el centro de esta cuenca hidrológica se encuentra la zona conurbada de los municipios de SLP y SGS que, en conjunto con los municipios de Mexquitic de Carmona, Cerro de San Pedro y Zaragoza, reúnen una población total de 1122502 (INEGI, 2010), y dependen del suministro del agua subterránea en un $95 \%$ para sus distintos usos (COTAS y CONAGUA, 2005).

La zona de estudio tiene un clima templado con verano cálido semiárido. La precipitación media anual es de $402.6 \mathrm{~mm}$ y una temperatura media anual de $17.5^{\circ} \mathrm{C}$. La evaporación potencial media anual es de $2038.7 \mathrm{~mm}$.

\subsection{Marco Hidrogeológico}

En esta zona se ha identificado la presencia de dos acuíferos denominados tradicionalmente como somero (granular) y profundo (volcánico fracturado) (CardonaBenavides, 1990; Carrillo-Rivera et al., 1992; CarrilloRivera et al., 1996) separados por un estrato de arena fina compacta de baja conductividad hidráulica. Como parte del acuífero granular se encuentra un acuífero de tipo colgado, siendo éste quien recibe directamente los efectos de la actividad humana.

\subsubsection{Acuifero colgado}

El acuífero colgado es muy superficial, tiene una profundidad de apenas 40 metros aproximadamente (COTAS y CONAGUA, 2005) y presenta una extensión limitada (en la zona urbana). Es un estrato de baja conductividad hidráulica, se recarga a partir de la precipitación en el valle y su comportamiento es muy dinámico. Tiene recarga inducida de tipo difusa (ocasionalmente por la fuga de agua potable y drenaje) y por retornos de riego.

Los flujos por retorno de riego son responsables del deterioro de la calidad del agua subterránea en un gran número de países, en particular en regiones semiáridas y áridas (UNESCO, 2004).

Debido a la poca profundidad a la que se encuentra, y que gran parte de los cultivos se irrigan con aguas negras municipales sin tratamiento, se ha detectado la presencia de contaminación en el agua subterránea por metales, contaminantes orgánicos, inorgánicos y bacteriológicos (Carrillo-Rivera y Armienta, 1989; Carrillo-Rivera et al., 2008; Moran-Ramírez, 2010; IPICYT, 2010).

El material que compone este acuífero tiene variaciones texturales, de tal manera que hacia la SSM predominan conglomerados inmersos en un matriz areno arcillosa y hacia el noreste del valle este material se adelgaza y predominan limos y arenas.

La dirección de flujo del acuífero colgado va de Suroeste a Noreste. Su recarga depende de las condiciones como la precipitación evapotraspiración, escurrimiento, vegetación, presencia de capa de suelo, pendiente del terreno y permeabilidad de las rocas. La principal recarga en el acuífero se efectúa en el flanco oriente del VSLP, así como al norte de la SSM hacia el Valle de Escalerillas (Ramos-Leal et al., 2007). Hacia la localidad de La Palma, se ubica el límite del acuífero colgado, en esta región el material granular se acuña y su contacto con el material volcánico fracturado es más próximo, por lo cual el flujo de éste puede tener comunicación hidráulica con el acuífero del medio granular.

\subsubsection{Acuifero granular}

El acuífero somero o granular que rellenó las fosas tectónicas ha sido el más explotado. Las estructuras geológicas, como las fallas, controlan la distribución y espesor de esta unidad acuífera; está compuesto principalmente por materiales clásticos del Cuaternario. El acuífero es actualmente explotado por pozos que alcanzan profundidades de hasta 350 metros de material sedimentario. Su límite superior se encuentra aproximadamente de 100 a 150 metros de profundidad. Está confinado en el centro del valle por una capa sedimentaria poco permeable. Su espesor va de 100 a 200 metros (IPICYT, 2007).

\subsubsection{Acuifero profundo}

El acuífero profundo, formado por roca volcánica fracturada, tiene una distribución irregular debido a un sistema de fosas y pilares en el valle. Lo limita por un lado la SSM, la cual, aunque está formada por este mismo material, en la sierra las fracturas han sido selladas por actividad hidrotermal, dando condiciones de baja permeabilidad. Por el otro lado lo limita la SA, cuyo origen es roca caliza de muy baja permeabilidad. Ambas fronteras tienen capacidad reducida para transmitir y almacenar agua por lo que restringen la recarga lateral.

Las unidades geológicas que constituyen el acuífero profundo tienen una distribución espacial más extensa que los límites de la cuenca superficial. Es de tipo confinado en la mayor parte de la porción plana del VSLP. La profundidad a la superficie potenciométrica es mayor a $150 \mathrm{~m}$, dependiendo de la ubicación dentro la planicie. La mayoría de los pozos perforados en este acuífero, tienen profundidades del orden de 350-450 m; aunque existen algunos de 800-1000 metros. Algunos pozos pueden atravesar 100-300 m de tobas e ignimbritas interestratificadas con el relleno granular, o alcanzar las rocas volcánicas que subyacen el relleno de la fosa tectónica.

\section{Metodología}

La campaña de muestreo hidrogeoquímico se realizó en octubre de 2008 con 47 muestras dentro del VSLP, 3 de ellas corresponden a manantiales y el resto a norias. El muestreo se realizó en el acuífero colgado a una profundidad 
promedio de $8.6 \mathrm{~m}$, teniendo un mínimo de $0.3 \mathrm{~m}$ y un máximo de $28 \mathrm{~m}$.

Los parámetros físico-químicos como temperatura, $\mathrm{pH}$, alcalinidad, conductividad eléctrica, sólidos totales disueltos y potencial redox fueron medidos en el sitio de muestreo. Las muestras tomadas para cationes y elementos traza se acidificaron con $\mathrm{HNO}_{3}$ concentrado para llevarlo a un $\mathrm{pH}<2$. A las muestras para aniones no se les realizó este procedimiento. La preservación del total de las muestras se dio a una temperatura menor a $4{ }^{\circ} \mathrm{C}$.

Los iones mayores y elementos traza fueron enviados a laboratorio de Geociencias de la Universidad Nacional Autónoma de México, los cuales fueron determinados por
ICPOES, los aniones (sulfatos y cloruros) por colorimetría en el IPICYT y la alcalinidad por titulación en campo. El balance iónico de los datos químicos tuvo un error menor al $10 \%$. Los datos de nitratos $\left(\mathrm{NO}_{3}^{-1}\right)$, sulfatos $\left(\mathrm{SO}_{4}^{-2}\right)$, cloruros $\left(\mathrm{Cl}^{-}\right)$, conductividad eléctrica $(\mathrm{CE})$, grasas y aceites (GA), coliformes totales (CT), fluoruros $\left(\mathrm{F}^{-}\right)$, arsénico $(\mathrm{As})$, estroncio $(\mathrm{Sr})$, bario $(\mathrm{Ba})$, hierro $(\mathrm{Fe})$, níquel $(\mathrm{Ni})$, aluminio $(\mathrm{Al})$, cobre $(\mathrm{Cu})$, mercurio $(\mathrm{Hg})$, plata $(\mathrm{Ag})$, plomo $(\mathrm{Pb})$, manganeso $(\mathrm{Mn})$, zinc $(\mathrm{Zn})$, cromo $(\mathrm{Cr})$, boro (B), cobalto (Co), cadmio (Cd), rubidio ( $\mathrm{Rb}$ ) y litio (Li) del agua subterránea fueron usados en diagramas distribución espacial para evaluar su comportamiento en el VSLP (Tabla1).

Tabla 1. Parámetros fisicoquímicos medidos en el acuífero colgado del Valle de San Luis Potosí.

\begin{tabular}{|c|c|c|c|c|c|c|c|c|c|c|c|c|c|c|c|c|c|c|}
\hline Punto & $X$ & $\mathrm{Y}$ & $\mathrm{pH}$ & $\begin{array}{c}\mathrm{Eh} \\
(\mathrm{mV})\end{array}$ & $\begin{array}{c}\mathrm{Na} \\
(\mathrm{ppm})\end{array}$ & $\begin{array}{c}\mathrm{K} \\
(\mathrm{ppm})\end{array}$ & $\begin{array}{c}\mathrm{Ca} \\
(\mathrm{ppm})\end{array}$ & $\begin{array}{c}\mathrm{Mg} \\
(\mathrm{ppm})\end{array}$ & $\begin{array}{l}\mathrm{NO}_{3} \\
(\mathrm{ppm})\end{array}$ & $\begin{array}{c}\mathrm{SO}_{4} \\
(\mathrm{ppm})\end{array}$ & $\begin{array}{l}\mathrm{HCO}_{3} \\
(\mathrm{ppm})\end{array}$ & $\begin{array}{c}\mathrm{Cl} \\
(\mathrm{ppm})\end{array}$ & $\begin{array}{c}\mathrm{CE} \\
(\mu \mathrm{S} / \mathrm{cm})\end{array}$ & $\begin{array}{c}\mathrm{CT} \\
(\mathrm{UFC})\end{array}$ & $\begin{array}{c}\text { GA } \\
(\mathrm{ppm})\end{array}$ & $\begin{array}{c}\mathrm{F} \\
(\mathrm{ppb})\end{array}$ & $\begin{array}{c}\mathrm{Hg} \\
(\mathrm{ppb})\end{array}$ & $\begin{array}{c}\mathrm{Ba} \\
(\mathrm{ppb})\end{array}$ \\
\hline 1 & 306326 & 2451142 & 6.7 & 63 & 89 & 40.2 & 215 & 25.2 & 25 & 276 & 270 & 126 & 126000 & 13.08 & 42.93 & 0.4 & 1.28 & 52.16 \\
\hline 3 & 306017 & 2452049 & 6.7 & 226 & 58 & 40.9 & 207 & 24.2 & 2 & 40 & 510 & 114.4 & 114400 & 9.17 & 59.58 & 0.54 & 0.27 & 236.61 \\
\hline 4 & 305308 & 2452724 & 6.3 & 62 & 102 & 35.2 & 299 & 29.2 & 8 & 120 & 474 & 212.8 & 212800 & 2.72 & 87.75 & 0.58 & 1.04 & 109.72 \\
\hline 5 & 306154 & 2453563 & 6.5 & 0.68 & 81 & 31.7 & 247 & 19.3 & 7 & 60 & 435 & 131.2 & 131200 & 11.99 & 10.05 & 0.64 & 1.18 & 178.04 \\
\hline 7 & 306481 & 2453867 & 7.1 & 98 & 140 & 21 & 136 & 6.2 & 5 & 52 & 315 & 134 & 134000 & 6.53 & 83.38 & 0.1 & 0.41 & 75.26 \\
\hline 8 & 305348 & 2453467 & 6.6 & 134 & 78 & 37.1 & 209 & 25.5 & 7 & 145 & 276 & 134.4 & 134400 & 4.49 & 166.18 & 0.07 & 0.83 & 68.25 \\
\hline 9 & 307305 & 2448872 & 6.9 & 0.58 & 126 & 22.6 & 89 & 6.2 & 4 & 52 & 345 & 73.2 & 73200 & 33.96 & 89.95 & 0.87 & 0.42 & 15.49 \\
\hline 10 & 305791 & 2443680 & 6.2 & 130 & 115 & 17.4 & 66 & 8 & 9 & 115 & 186 & 46.4 & 46400 & 2.42 & 285.65 & 0.83 & 0.52 & 82.14 \\
\hline 11 & 305957 & 2443942 & 6.1 & 138 & 191 & 37.1 & 153 & 17.5 & 24 & 240 & 225 & 160 & 160000 & 3.56 & 406.81 & 0.6 & 0.33 & 69.59 \\
\hline 12 & 306134 & 2444605 & 6.8 & 100 & 38 & 17.4 & 110 & 10.7 & 6 & 80 & 111 & 78 & 78000 & 10.81 & 3.98 & 0.6 & 0.38 & 52.39 \\
\hline 13 & 305642 & 2444615 & 6.7 & 5.17 & 81 & 28.7 & 91 & 11.8 & 8 & 140 & 135 & 72 & 72000 & 3.36 & 46.39 & 0.66 & 0.34 & 114.8 \\
\hline 14 & 305450 & 2444826 & 5.7 & 177 & 237 & 83.3 & 405 & 59.8 & 54 & 250 & 36 & 152.8 & 152800 & 3.96 & 13.72 & 1 & 0.14 & 73.93 \\
\hline 16 & 302758 & 2460264 & 6.2 & 148 & 24 & 22.8 & 90 & 9.4 & 6 & 60 & 210 & 38 & 38000 & 1.67 & 70.05 & 0.17 & 0.56 & 167.09 \\
\hline 17 & 306303 & 2459562 & 6.2 & 212 & 38 & 26.4 & 89 & 8.6 & 23 & 92 & 120 & 44 & 44000 & 1.39 & 202.51 & 0.08 & 0.93 & 81.47 \\
\hline 18 & 307442 & 2458220 & 6.5 & 191 & 61 & 29.8 & 135 & 15.9 & 27 & 56 & 330 & 77.6 & 77600 & 5.29 & 94.35 & 0.1 & 0.43 & 161.01 \\
\hline 19 & 305507 & 2456042 & 6.1 & 156 & 45 & 29.9 & 189 & 18.6 & 16 & 196 & 225 & 100 & 100000 & 1.77 & 74.55 & 0.19 & 1.4 & 60.96 \\
\hline 20 & 303754 & 2457371 & 6.4 & 161 & 53 & 31.8 & 255 & 24.2 & 52 & 84 & 300 & 196 & 196000 & 13.79 & 68.8 & 0.01 & 1.57 & 122.21 \\
\hline 21 & 300041 & 2457828 & 6.4 & 147 & 61 & 22 & 175 & 15.3 & 23 & 76 & 330 & 104 & 104000 & 24.03 & 207.75 & 0.11 & 0.75 & 275.74 \\
\hline 22 & 298584 & 2454733 & 6.4 & 158 & 81 & 46.6 & 134 & 21.8 & 24 & 96 & 285 & 91 & 91000 & 16.71 & 1.36 & 0.67 & 0.49 & 48.9 \\
\hline 23 & 298654 & 2457394 & 6.6 & 326 & 43 & 38.3 & 165 & 16 & 6 & 14 & 450 & 70 & 70000 & 2.63 & 416.23 & 0.5 & 0.4 & 297.31 \\
\hline 24 & 300910 & 2452763 & 6.8 & 259 & 95 & 22.3 & 75 & 8.2 & 12 & 46 & 225 & 64 & 64000 & 1.75 & 5.24 & 0.63 & 0.36 & 53.85 \\
\hline 25 & 301150 & 2452968 & 6.7 & 241 & 174 & 40.2 & 130 & 18.8 & 32 & 114 & 360 & 120 & 120000 & 2.49 & 5.03 & 0.35 & 0.51 & 50.46 \\
\hline 26 & 299511 & 2452943 & 6.8 & 342 & 200 & 91 & 210 & 29.8 & 45 & 196 & 411 & 164 & 164000 & 20.49 & 80.73 & 0.52 & 0.37 & 116.53 \\
\hline 27 & 299108 & 2453004 & 6.4 & 328 & 165 & 70.9 & 205 & 28.8 & 34 & 204 & 381 & 218 & 218000 & 3.78 & 22.72 & 0.44 & 0.44 & 62.4 \\
\hline 28 & 296606 & 2453527 & 6.7 & 334 & 64 & 85.8 & 167 & 19.4 & 14 & 200 & 345 & 65 & 65000 & 4.91 & 30.26 & 0.61 & 0.52 & 92.09 \\
\hline 29 & 292058 & 2450938 & 7.3 & 44 & 81 & 39.7 & 175 & 33.8 & 12 & 194 & 513 & 52 & 52000 & 25.88 & 78.74 & 0.35 & $<0.1$ & 136.63 \\
\hline 30 & 296662 & 2450956 & 6.4 & 324 & 58 & 54.6 & 92 & 11.3 & 22 & 50 & 243 & 76 & 76000 & 2.16 & 88.59 & 0.4 & 0.27 & 88.89 \\
\hline 33 & 295218 & 2453336 & 6.8 & 275 & 75 & 64.8 & 73 & 8.6 & 13 & 44 & 270 & 67.2 & 67200 & 18.48 & 4.4 & 0.57 & 0.57 & 72.86 \\
\hline 34 & 293326 & 2455218 & 6.5 & 283 & 68 & 15.4 & 68 & 5.6 & 10 & 56 & 210 & 54 & 54000 & 1.68 & 10.37 & 0.3 & 0.43 & 72.46 \\
\hline 35 & 293959 & 2452014 & 6.6 & 28.6 & 66 & 46 & 71 & 13.1 & 31 & 94 & 240 & 52 & 52000 & 1.96 & 70.68 & 0.48 & 1.68 & 76.4 \\
\hline 36 & 291145 & 2454412 & 6.1 & 290 & 36 & 25.4 & 54 & 12.2 & 18 & 110 & 120 & 18.4 & 18400 & 16.67 & 3.77 & 0.38 & 0.75 & 96.03 \\
\hline 37 & 295658 & 2455196 & 6.8 & 134 & 153 & 30.7 & 57 & 8.1 & 12 & 126 & 270 & 64 & 64000 & 27.85 & 423.98 & 0.52 & 0.96 & 37.44 \\
\hline 38 & 287239 & 2452508 & 6.1 & 296 & 39 & 45.5 & 76 & 15 & 10 & 176 & 120 & 43.2 & 43200 & 24.32 & 2.83 & 0.33 & 0.85 & 87.62 \\
\hline 39 & 289442 & 2454311 & 6.2 & 297 & 88 & 35.4 & 90 & 11.8 & 26 & 146 & 138 & 104 & 104000 & 12.15 & 135.18 & 0.35 & 1.14 & 56.04 \\
\hline 40 & 295454 & 2446242 & 5.1 & 344 & 34 & 10.9 & 14 & 2.7 & 9 & 88 & 30 & 10.4 & 10400 & 6.52 & 57.17 & 0.01 & 0.47 & 33 \\
\hline 41 & 296709 & 2447189 & 6.1 & 293 & 36 & 25.8 & 55 & 11.5 & 13 & 144 & 120 & 21.6 & 21600 & 8.12 & 13.93 & 0.19 & 0.7 & 88.35 \\
\hline 42 & 297933 & 2445273 & 6 & 299 & 39 & 29.1 & 85 & 24.1 & 6 & 212 & 102 & 28.8 & 28800 & 12.86 & 2.51 & 0.17 & 0.23 & 50.85 \\
\hline 43 & 299953 & 2444649 & 5.5 & 265 & 19 & 10.8 & 21 & 3.9 & 4 & 52 & 40 & 10.8 & 10800 & 11.54 & 40.84 & 0.03 & 0.47 & 73.46 \\
\hline 44 & 303907 & 2432230 & 6.3 & 45 & 21 & 5.9 & 9 & 1.2 & 9 & 20 & 40 & 8.4 & 8400 & 7.09 & 109.63 & 0.01 & 0.13 & 7.99 \\
\hline 45 & 304071 & 2434080 & 6.6 & 73 & 51 & 31 & 57 & 12.5 & 30 & 90 & 60 & 72.8 & 72800 & 12.13 & 29.21 & 0.2 & 0.29 & 111.12 \\
\hline 46 & 302770 & 2438427 & 5.9 & 115 & 21 & 15.8 & 20 & 2.9 & 4 & 48 & 60 & 10.4 & 10400 & 13.79 & 60.1 & 0.01 & 0.39 & 40.58 \\
\hline 47 & 303005 & 2441654 & 5.7 & 140 & 29 & 18.4 & 26 & 4.4 & 15 & 60 & 40 & 16.4 & 16400 & 10.9 & 165.45 & 0.01 & 0.69 & 80.67 \\
\hline
\end{tabular}


Tabla 1. Parámetros fisicoquímicos medidos en el acuífero colgado del Valle de San Luis Potosí (continuación).

\begin{tabular}{|c|c|c|c|c|c|c|c|c|c|c|c|c|c|c|c|c|c|}
\hline Punto & $X$ & $\mathrm{Y}$ & $\begin{array}{c}\mathrm{Sr} \\
(\mathrm{ppb})\end{array}$ & $\begin{array}{c}\mathrm{Cd} \\
(\mathrm{ppb})\end{array}$ & $\begin{array}{c}\mathrm{Pb} \\
(\mathrm{ppb})\end{array}$ & $\begin{array}{c}\mathrm{Ag} \\
(\mathrm{ppb})\end{array}$ & $\begin{array}{c}\mathrm{Rb} \\
(\mathrm{ppb})\end{array}$ & $\begin{array}{c}\text { Co } \\
(\mathrm{ppb})\end{array}$ & $\begin{array}{c}\mathrm{Cu} \\
(\mathrm{ppb})\end{array}$ & $\begin{array}{c}\mathrm{Fe} \\
(\mathrm{ppb})\end{array}$ & $\begin{array}{c}\text { As } \\
(\mathrm{ppb})\end{array}$ & $\begin{array}{c}\mathrm{Li} \\
(\mathrm{ppb})\end{array}$ & $\mathrm{Ni}(\mathrm{ppb})$ & $\begin{array}{c}\mathrm{Mn} \\
(\mathrm{ppb})\end{array}$ & $\begin{array}{c}\mathrm{Cr} \\
(\mathrm{ppb})\end{array}$ & $\begin{array}{c}\mathrm{Zn} \\
(\mathrm{ppb})\end{array}$ & $\begin{array}{c}\mathrm{Al} \\
(\mathrm{ppb})\end{array}$ \\
\hline 1 & 306326 & $2 \mathrm{E}+06$ & 1126.66 & 0.82 & 2.64 & 0.13 & 106.63 & 0.16 & 0.24 & 33.37 & 12.6 & 6.53 & 7.55 & 1.16 & 0.59 & 3.34 & $<0.1$ \\
\hline 2 & 305775 & $2 \mathrm{E}+06$ & 1419.26 & 0.55 & 2.86 & $<0.1$ & 98.75 & 0.25 & 2.73 & 39.23 & 20.21 & 4.18 & 4.2 & 2.81 & 0.5 & 11.81 & 0.96 \\
\hline 3 & 306017 & $2 \mathrm{E}+06$ & 1241.37 & 0.29 & 0.54 & $<0.1$ & 109.02 & 0.37 & $<0.1$ & 52.86 & 10.45 & 5.18 & 3.42 & 800.54 & 0.39 & 2.84 & $<0.1$ \\
\hline 4 & 305308 & $2 \mathrm{E}+06$ & 1576.74 & 2.51 & 1.91 & 0.19 & 104.13 & 0.36 & 0.25 & 46.06 & 10.25 & 4.59 & 5.07 & 0.99 & 0.55 & 18.71 & 2.46 \\
\hline 5 & 306154 & $2 \mathrm{E}+06$ & 1111.1 & 0.22 & 1.2 & 3.07 & 85.48 & 0.26 & 1.14 & 29.6 & 10.41 & 3.89 & 3.02 & 26.3 & 0.29 & 4.42 & $<0.1$ \\
\hline 6 & 305982 & $2 \mathrm{E}+06$ & 904.44 & 0.35 & 0.47 & 1.74 & 79.45 & 0.19 & $<0.1$ & 22.12 & 7.77 & 3.88 & 2.47 & 2.68 & 0.4 & 2.71 & $<0.1$ \\
\hline 7 & 306481 & $2 \mathrm{E}+06$ & 391.95 & 0.13 & 0.72 & $<0.1$ & 37.08 & 0.47 & 0.72 & 21.16 & 7.5 & 10.5 & 2.08 & 0.68 & 0.19 & 3.93 & $<0.1$ \\
\hline 8 & 305348 & $2 \mathrm{E}+06$ & 1403.7 & 0.59 & 1 & $<0.1$ & 103.93 & 0.36 & 1 & 29.97 & 8.04 & 5.48 & 3.4 & 43.97 & 0.35 & 29.73 & $<0.1$ \\
\hline 9 & 307305 & $2 \mathrm{E}+06$ & 161.27 & 5.86 & 21.86 & $<0.1$ & 41.52 & 0.43 & 62.67 & 77.12 & 9.84 & 90.36 & 15.45 & 179.61 & 1.23 & 301.9 & 27.97 \\
\hline 10 & 305791 & $2 \mathrm{E}+06$ & 314.76 & 0.87 & 3.13 & $<0.1$ & 59.43 & 0.36 & 4.33 & 36.47 & 11.87 & 9.13 & 12.08 & 7.53 & 0.6 & 19.51 & 54.53 \\
\hline 11 & 305957 & $2 \mathrm{E}+06$ & 614.66 & 0.27 & 0.51 & $<0.1$ & 105.05 & 4.84 & 0.1 & 25.43 & 9.89 & 14.88 & 5.95 & 3.78 & 0.41 & 4.26 & $<0.1$ \\
\hline 12 & 306134 & $2 \mathrm{E}+06$ & 429.47 & 0.43 & 2.18 & $<0.1$ & 43.91 & 0.27 & 1.78 & 31.25 & 63.72 & 6.18 & 2.86 & 86.65 & 0.14 & 35.95 & 3.19 \\
\hline 13 & 305642 & $2 \mathrm{E}+06$ & 449.4 & 1.88 & 1.69 & $<0.1$ & 81.98 & 0.52 & 0.74 & 20.55 & 4.78 & 4.44 & 24.7 & 2.43 & 161.91 & 14.43 & 8.38 \\
\hline 16 & 302758 & $2 \mathrm{E}+06$ & 451.25 & 1.45 & 0.79 & $<0.1$ & 66.5 & $<0.1$ & $<0.1$ & 21.26 & 4.32 & 3.99 & 1.29 & 1.18 & 0.37 & 2.32 & $<0.1$ \\
\hline 17 & 306303 & $2 \mathrm{E}+06$ & 492.27 & 2.71 & 2.85 & 0.44 & 71.18 & 0.1 & 0.55 & 24.66 & 7.57 & 4.31 & 1.61 & 1.19 & 0.49 & 8.5 & 7.3 \\
\hline 18 & 307442 & $2 \mathrm{E}+06$ & 834.45 & 0.25 & 0.96 & 0.28 & 71.43 & 0.2 & 0.68 & 26.33 & 7.85 & 5.75 & 2.61 & 4.33 & 0.23 & 5.22 & 2.09 \\
\hline 19 & 305507 & $2 \mathrm{E}+06$ & 978.9 & 0.44 & 0.77 & $<0.1$ & 83.95 & 0.15 & $<0.1$ & 21.5 & 5.55 & 4.74 & 2.31 & 1.8 & 0.12 & 2.99 & $<0.1$ \\
\hline 20 & 303754 & $2 \mathrm{E}+06$ & 1167.49 & 1.04 & 2.09 & 1.43 & 98.86 & 0.47 & 1.33 & 29.77 & 7.09 & 3.08 & 4.38 & 1.49 & 0.57 & 187.66 & $<0.1$ \\
\hline 21 & 300041 & $2 \mathrm{E}+06$ & 975.01 & 0.21 & 0.49 & 0.1 & 64.24 & 0.28 & 0.29 & 26.45 & 5.05 & 4.25 & 3.41 & 3.31 & 0.14 & 3.25 & $<0.1$ \\
\hline 22 & 298584 & $2 \mathrm{E}+06$ & 660.54 & 0.84 & 1.45 & $<0.1$ & 165.76 & 0.21 & 0.86 & 26.5 & 5.25 & 5.47 & 2.22 & 0.99 & 0.29 & 8.05 & 55.89 \\
\hline 23 & 298654 & $2 \mathrm{E}+06$ & 1140.27 & 0.23 & 0.6 & $<0.1$ & 72.52 & 0.63 & $<0.1$ & 29.8 & 13.44 & 4.31 & 4.25 & 21.03 & 0.2 & 5.15 & 34.1 \\
\hline 24 & 300910 & $2 \mathrm{E}+06$ & 370.37 & 0.21 & 0.77 & $<0.1$ & 59.8 & 0.1 & 221.8 & 19.56 & 11.62 & 3.6 & 1.68 & 0.6 & 0.33 & 8.67 & 6.13 \\
\hline 25 & 301150 & $2 \mathrm{E}+06$ & 768.64 & 0.31 & 0.44 & 0.1 & 95.05 & 0.17 & $<0.1$ & 18.35 & 5.6 & 4.43 & 1.95 & 0.89 & 0.18 & 2.25 & $<0.1$ \\
\hline 26 & 299511 & $2 \mathrm{E}+06$ & 1293.86 & 3.22 & 3.01 & $<0.1$ & 288.11 & 2.9 & 2.47 & 40.57 & 48.93 & 30.95 & 9.46 & 970.38 & 1.4 & 15.43 & 8.36 \\
\hline 27 & 299108 & $2 \mathrm{E}+06$ & 1164.57 & 0.27 & 0.51 & $<0.1$ & 185.67 & 0.3 & $<0.1$ & 24.55 & 64.09 & 6.19 & 2.69 & 9.84 & 22.53 & 1.09 & $<0.1$ \\
\hline 28 & 296606 & $2 \mathrm{E}+06$ & 856.9 & 0.96 & 1.1 & $<0.1$ & 203.81 & 0.21 & $<0.1$ & 25.91 & 40.07 & 5.36 & 2.81 & 2.13 & 0.31 & 4.61 & $<0.1$ \\
\hline 29 & 292058 & $2 \mathrm{E}+06$ & 1165.54 & 0.27 & 1.25 & $<0.1$ & 101.96 & 0.27 & $<0.1$ & 29.93 & 10.86 & 6.91 & 4.24 & 1859.66 & 0.63 & 2.51 & 3.53 \\
\hline 30 & 296662 & $2 \mathrm{E}+06$ & 443.47 & 3.84 & 1.38 & 0.3 & 148.98 & 2.52 & 0.05 & 32.64 & 44.64 & 3.41 & 3.7 & 1.48 & 0.45 & 3.9 & 14.37 \\
\hline 33 & 295218 & $2 \mathrm{E}+06$ & 447.55 & 1.72 & 0.49 & 0.81 & 105.22 & 0.22 & $<0.1$ & 24.3 & 22.46 & 5.42 & 2.34 & 0.59 & 6.52 & 7.33 & $<0.1$ \\
\hline 34 & 293326 & $2 \mathrm{E}+06$ & 317.58 & 0.81 & 1.64 & 0.17 & 42.45 & 0.09 & 2.58 & 33.36 & 10.45 & 5.07 & 1.27 & 1.12 & 0.35 & 15.44 & 1.25 \\
\hline 35 & 293959 & $2 \mathrm{E}+06$ & 498.68 & 2.44 & 0.96 & 1.13 & 91.99 & 0.11 & $<0.1$ & 24.48 & 9.34 & 2.53 & 1.3 & 0.62 & 0.97 & 4.54 & 0.63 \\
\hline 36 & 291145 & $2 \mathrm{E}+06$ & 327.5 & 0.27 & 0.4 & 0.34 & 78.22 & $<0.1$ & $<0.1$ & 21.66 & 2.51 & 10.59 & 1 & 0.91 & 0.1 & 10.29 & $<0.1$ \\
\hline 37 & 295658 & $2 \mathrm{E}+06$ & 383.78 & 0.47 & 1.26 & 0.13 & 64.42 & 0.1 & 0.25 & 119.87 & 17.92 & 4.9 & 1.53 & 20.7 & 0.2 & 3 & 3.28 \\
\hline 38 & 287239 & $2 \mathrm{E}+06$ & 405.36 & 0.32 & 1.17 & 0.23 & 90.12 & $<0.1$ & 0.29 & 20.78 & 74.1 & 3.21 & 1.35 & 4.33 & 0.16 & 34.91 & 1.12 \\
\hline 39 & 289442 & $2 \mathrm{E}+06$ & 431.42 & 1.34 & 1.79 & 0.83 & 71.98 & 0.23 & 0.71 & 22.72 & 18.89 & 5.14 & 1.94 & 0.87 & 0.24 & 10.1 & 10.37 \\
\hline 40 & 295454 & $2 \mathrm{E}+06$ & 78.61 & 0.46 & 1.6 & 0.43 & 24.36 & 0.13 & 0.17 & 15.29 & 12.29 & 9.33 & 1.22 & 5.93 & $<0.1$ & 4.18 & 9.18 \\
\hline 41 & 296709 & $2 \mathrm{E}+06$ & 294.54 & 0.56 & 1.15 & $<0.1$ & 54.49 & 0.14 & 3.85 & 46.07 & 6.83 & 4.36 & 1.63 & 24.04 & 0.13 & 4.38 & $<0.1$ \\
\hline 42 & 297933 & $2 \mathrm{E}+06$ & 508.21 & 1.63 & 0.69 & $<0.1$ & 61.2 & 0.11 & $<0.1$ & 13.39 & 5.71 & 11.67 & 2.07 & 16.68 & 0.2 & 5.77 & $<0.1$ \\
\hline 43 & 299953 & $2 \mathrm{E}+06$ & 133.86 & 0.27 & 0.48 & $<0.1$ & 23.99 & 0.1 & $<0.1$ & 17.27 & 4.29 & 7.81 & 1.04 & 5.97 & $<0.1$ & 3.13 & $<0.1$ \\
\hline 44 & 303907 & $2 \mathrm{E}+06$ & 30.71 & 0.56 & 2.4 & $<0.1$ & 32.23 & $<0.1$ & 0.83 & 25.15 & 11.95 & 6.7 & 0.5 & 4.45 & 0.33 & 5.69 & 13.75 \\
\hline 45 & 304071 & $2 \mathrm{E}+06$ & 303.97 & 1.98 & 27.9 & $<0.1$ & 47.46 & 0.14 & 10.85 & 13.1 & 12.91 & 8.55 & 2.19 & 4.39 & 0.36 & 38.17 & 18.22 \\
\hline 46 & 302770 & $2 \mathrm{E}+06$ & 94.77 & 2.34 & 6.65 & 0.11 & 31.01 & 0.1 & 3.05 & 143.3 & 9.53 & 10.16 & 1.46 & 4.05 & 0.53 & 15.2 & 540.97 \\
\hline 47 & 303005 & $2 \mathrm{E}+06$ & 139.5 & 0.86 & 5.62 & 0.29 & 33.08 & 0.11 & 2.6 & 73.33 & 5.45 & 9.45 & 1.47 & 3.62 & 0.36 & 11.28 & 218.47 \\
\hline
\end{tabular}

\section{Resultados y Discusión}

\subsection{Evolución del uso de suelo en el Valle de San Luis Potosí}

Como consecuencia de los primeros asentamientos humanos en el valle a mediados del siglo XVI, se da inicio a los dos primeros usos de suelo (urbano y minero) en el actual VSLP. El uso de suelo urbano generó agua residuales domésticas que eran vertidas en corrientes naturales y canales abiertos.

Aunque la extracción de minerales se desarrollaba en el Cerro de San Pedro, el procesado de los minerales se llevaba a cabo en patios de beneficio en el centro de la actual ciudad de SLP (Figura 2), debido a que allí era donde existían los pequeños manantiales y corrientes como la del Río de Santiago. Los residuos que se generaban de esta actividad eran depositados en patios de beneficio dentro de la zona urbana sin algún tipo de manejo apropiado (Figura 2), generando la lixiviación de los residuos mineros (KeiserSchlittler, 1992; Galván-Arellano, 1999; IMPLAN, 2009). La ciudad en constante crecimiento desplazó esta actividad 
hacia la periferia, sin embargo, sus residuos quedaron en un principio abandonados, luego sepultados y/o distribuidos en la ciudad para ser ocupados principalmente como relleno de calles.

La agricultura desarrollada de manera importante en el siglo XVII, a través de pequeños huertos familiares (Figura 2 ), en sus inicios no representó un agente de alto impacto, sin embargo, se convirtió en un factor de alteración cuando comenzó a recibir las aguas residuales que generaba la ciudad. Un caso muy particular es el sitio conocido como Tanque Tenorio (ubicado al oriente de la ciudad) que originalmente era una depresión natural donde se formaba un cuerpo de agua intermitente. Desde los años setenta del siglo pasado se comenzó a usar para descargar aguas residuales industriales, y en menor medida domésticas, en forma continua (Medellín-Milán, 2003). Estas aguas han sido aprovechadas desde entonces para riego en la zona agrícola y por su origen impactan al acuífero colgado (Moran-Ramírez, 2010; IPICYT, 2010).

La industria surgió de manera importante en la segunda mitad del siglo XX, junto con éste, el crecimiento de la superficie urbana multiplicó su superficie rápidamente de 1760 hectáreas en 1960 a 14 mil hectáreas en el año 2000 (Figura 3), otro evento fue el auge de la diversificación e intensificación industrial (SEMARNAT, 2008).

La consecuencia inmediata del crecimiento industrial fue la instauración de nuevas áreas de vivienda para aquellas familias que llegaron a la ciudad en busca de fuentes de empleo. La mancha urbana se extendió principalmente sobre terrenos yermos y comenzó un desplazamiento importante de los cultivos periurbanos (Figura 3), generando aguas residuales domésticas que son utilizadas en la zona agrícola. En tanto que la industria genera aguas residuales industriales sin tratamiento y utilizadas en la agricultura.

El cambio de uso de suelo significó modificaciones al sistema acuífero en cantidad y calidad. De tal manera que la actividad agrícola demanda volúmenes de agua de forma cíclica, mientras que los nuevos usos del suelo (industrial, habitacional y comercial) demanda un volumen constante y abasto permanente (SEMARNAT, 2008).

Un indicador del impacto del cambio de uso de suelo es el origen del agua para abastecimiento de la ciudad. En 1960, el 59\% del agua era de origen superficial y $41 \%$ subterráneo, pero para 2005 la proporción cambió drásticamente a $8 \%$ de origen superficial y $92 \%$ de origen subterráneo generando una dependencia creciente y acelerada del acuífero profundo (COTAS y CONAGUA, 2005).

En cuanto a calidad se refiere, en los primeros asentamientos humanos se utilizaba agua superficial de buena calidad acumulada en los pequeños manantiales. Posteriormente, en la primera mitad del siglo XX, una parte de la población se abastecía directamente de norias que eran utilizadas simultáneamente para el riego de huertas y el uso doméstico (Sheridan-Prieto, 2001); sin embargo, los cambios en el uso del suelo extinguieron los huertos que existían dentro de la ciudad, originando una competencia por el agua de mejor calidad, convirtiendo a las norias en la fuente principal para la agricultura y las extracciones del acuífero profundo se destinaron principalmente a los usos doméstico, comercial e industrial.

En la actualidad, la agricultura recibe las descargas de aguas residuales domésticas e industriales que son conducidas por canales a cielo abierto contribuyendo con un impacto directo sobre el acuífero colgado (COTAS y CONAGUA, 2005; Moran-Ramírez, 2010).

\subsection{Fuentes Contaminantes del Agua Subterránea en el Valle de San Luis Potosí}

Aunque existe una gran cantidad de actividades humanas que generan cierta carga contaminante, un pequeño número de éstas se pueden considerar como tal (Ramos-Leal, 2002). Dependiendo de los mecanismos de control primario de cada actividad, éstas se pueden clasificar como fuentes potenciales y activas (Wilson, 1991), las primeras no están de hecho contaminando, pero representan un riesgo; las segundas son aquellas en las que los mecanismos de control primario han fallado y por lo tanto, representan una fuente contaminante activa.

Se considera como fuente de contaminación del acuífero al sitio que genera lixiviados o solutos y que al ser incorporados al sistema acuífero alteran la calidad natural del agua subterránea. La calidad del agua subterránea está definida por los valores de fondo de los elementos que de manera natural se incorporan al flujo subterráneo, estas concentraciones no necesariamente tienen que estar por debajo de la normatividad vigente para agua potable.

\subsection{Tipo de fuentes potenciales de contaminación}

En el Valle de San Luis Potosí las fuentes contaminantes pueden ser caracterizadas por su origen, geometría, actividad o temporalidad (Tablas 2, 3, 4 y 5).

\subsubsection{Origen}

La contaminación puede ser de tipo natural o antropogénica cuando está asociado a alguna actividad humana (Fetter, 1999).

El acuífero colgado puede ser categorizado como urbano debido a que una superficie importante del acuífero se encuentra debajo de los tres principales usos de suelo (urbano, industrial y agrícola), por lo tanto el origen de la contaminación está asociada a las actividades humanas y su condición estará directamente afectada por la dinámica y el tipo de crecimiento urbano (Peña, 2006).

Cuando la contaminación no está asociada con actividades humanas su origen es natural, como en el caso de la lixiviación de rocas y suelos (Fetter, 1999; ArmientaHernández et al., 1996; Robles y Armienta, 2000). En el caso del agua subterránea, para el acuífero profundo, en el área de estudio se han reportado niveles de flúor por encima de la norma (Carrillo-Rivera et al., 2002; COTAS y 
Tabla 2. Tipos de fuentes potencialmente contaminantes de acuerdo a su origen, geometría, actividad y temporalidad (Fetter, 1999).

\begin{tabular}{lccc}
\hline Origen & Natural & Antropogénico & \\
Geometría & Puntual & Lineal & Difusa \\
Actividad & Activa & Potencial & Inactiva \\
Temporalidad & Pulso & Intermitente & Permanente \\
\hline
\end{tabular}

CONAGUA, 2005; Ramos-Leal, 2002; Cardona-Benavides, 2007), cuyo origen es asociado con las rocas volcánicas que forman el acuífero.

En el caso del acuífero colgado los niveles de fluoruros son bajos con respecto a la NOM-127 SSAI-1994.

Las anomalías encontradas pueden ser causadas, en primer lugar, debido a la disposición de residuos de flúor y en segundo a talleres de soldadura, en donde se emplea como fundente (Bateman, 1974 ).

Las anomalías de As, Li y los valores de F- que sobrepasan la NOM-127 SSAI-1994 en el acuífero profundo están asociadas al medio geológico (COTAS y CONAGUA, 2005; Cardona-Benavides, 2007; Carrillo-Rivera et al., 2002), mientras que para el agua del acuífero colgado las concentraciones son bajas y las anomalías pueden estar asociadas a actividades antropogénicas.

\subsubsection{Geometría}

De acuerdo a su geometría, una fuente es puntual cuando, dependiendo de la escala a la que se trabaje, ésta puede ser representada por un punto en el espacio. En el área de estudio este tipo de contaminación es generada por los patios de beneficio minero en el siglo XVII en la zona urbana; en la actualidad algunos puntos en la zona industrial muestran este comportamiento.

Si la representación es una línea o una sucesión de puntos, será lineal, como en el caso los canales a cielo abierto en la zona agrícola de SGS y los ríos Santiago y Españita en la zona urbana.

Si la fuente se encuentra dispersa en la zona de estudio o si la generación de lixiviaciones tiene lugar en más de dos puntos, por su geometría se le considera como difusa. Este tipo de geometría es la presentada por $\mathrm{NO}_{3}^{-1}, \mathrm{SO}_{4}^{-2}, \mathrm{Cl}^{-}$y $\mathrm{CE}$ en la zona urbana y agrícola.

Otra fuente de contaminación, de tipo difusa, es generada por la dispersión de partículas atmosféricas, como las reportadas en la zona industrial; partículas con un metal pesado dominante (partículas ricas en hierro, cobre, plomo, arsénico, zinc, níquel, estaño, aluminio, molibdeno y bismuto), así como anomalías de partículas de fluorita, cuya abundancia se debe al manejo del mineral que es transportado hacia la zona industrial en donde se tritura y almacena al aire libre (Aragón-Piña et al., 2006). Otro tipo de partículas reportadas por estos mismos autores, son de cobre metálico, sulfatos de plomo y trióxido de arsénico, relacionadas a la zona minero-metalúrgica, ubicada al NW de la ciudad.

\subsubsection{Actividad}

En caso de existir evidencias de la generación de lixiviaciones será activa, como es el caso de los usos de suelo actuales en donde la contaminación es permanente; un ejemplo de este tipo de actividad es la zona agrícola de SGS, a través de los canales y parcelas de riego. En la zona industrial es por medio de sus descargas.

Cuando existen o pueden existir elementos suficientes para que se generen lixiviaciones en un futuro inmediato o a largo plazo, será contaminación de tipo potencial. En zonas urbanas los tiraderos clandestinos y la disposición de residuos de flúor.

Las fuentes inactivas siguen representando un riesgo potencial. Tal es el caso de los patios de beneficio minero del siglo XVII en la zona urbana (Figura 2) y el antiguo Tanque Tenorio (Keiser-Schlittler, 1992; Galván-Arellano, 1999; Medellín-Milán, 2003; IMPLAN, 2009).

\subsubsection{Temporalidad}

Cuando la generación de lixiviados es continua en el tiempo, se le definirá como permanente, como es el caso de los canales a cielo abierto en la zona agrícola.

Si los lixiviados solo están presentes cuando las condiciones de humedad en el sitio lo permiten (lluvias e incremento de humedad) será intermitente, como es el caso de los ríos Santiago y Españita. Otro caso son los residuos sólidos mineros sepultados en la zona urbana los cuales generan lixiviados en temporada de lluvias y, cuando el nivel freático se eleva, éstos son incorporados al acuífero.

Cuando los lixiviados ocurren en una ocasión, su carácter temporal será de pulso. Debido a que en el área de estudio el nivel freático está muy cercano a la superficie, el acuífero es muy vulnerable a la contaminación. Bajo estas condiciones en época de estiaje, los valores de contaminantes se incrementan y en época de lluvia son incorporados y diluidos en el acuífero.

Los cambios de uso de suelo en la zona de estudio y sus efectos se aprecian en la Figura 4, para el año 1989 la distribución de $\mathrm{NO}_{3}^{-1}$ era principalmente en la zona agrícola; para 1995, además del crecimiento del uso de suelo urbano, es apreciable el incremento de la marcha de este contaminante. En 2009 la distribución de $\mathrm{NO}_{3}^{-1}$, permanece en la zona agrícola además de encontrarse de manera importante en la zona urbana, como producto de los desechos humanos.

\subsubsection{Contaminación en zonas urbanas}

Entre las fuentes potenciales de contaminación que existen en la zona urbana y conurbada de SLP se encuentran panteones, jales mineros, descargas de aguas residuales, disposición de residuos sólidos (activos e inactivos), gasolineras y formaciones geológicas que aportan fluor a la región (Figura 1; Tabla 3). Derivado de estas fuentes potenciales de contaminación, en el acuífero colgado de la zona urbana se detectaron niveles importantes de $\mathrm{NO}_{3}^{-1}$, 


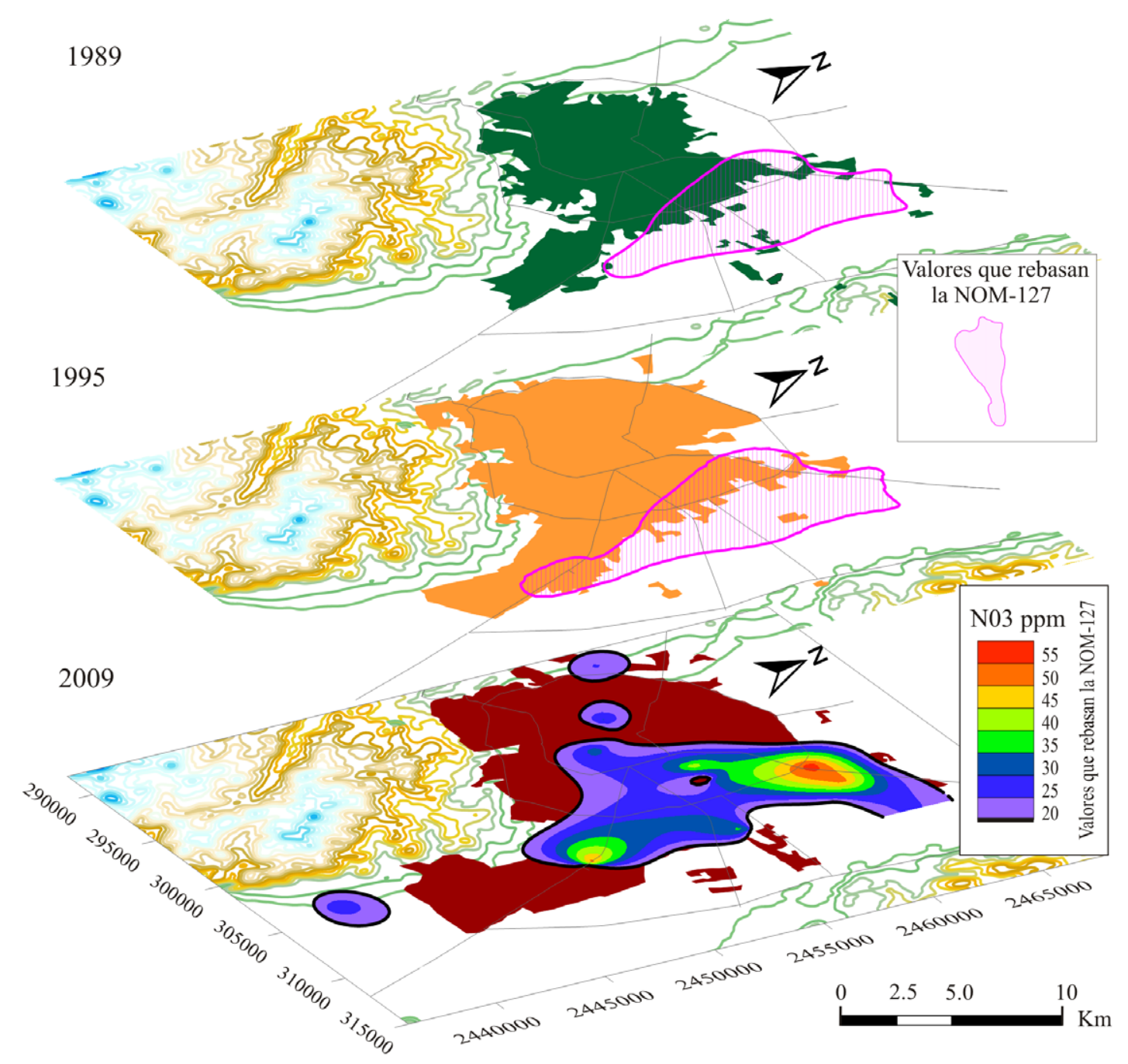

Figura 4. Distribución temporal de $\mathrm{NO}_{3}$ en la zona de estudio, basados en datos de Moran-Ramírez, 2010; COTAS y CONAGUA, 2005).

Tabla 3. Tipos de fuentes potencialmente contaminantes de acuerdo a su actividad, origen, geometría, temporalidad y tipo de soluto en la zona urbana de San Luis Potosí.

\begin{tabular}{|c|c|c|c|c|c|c|}
\hline Tipo de fuente & Actividad & Origen & Geometría & Temporalidad & Soluto & Uso de Suelo \\
\hline Zona urbana & Activa & Antropogénico & Difusa & Permanente & Nitratos, SDT. & Zona Urbana \\
\hline Panteones & Potencial & Antropogénico & Puntual & Intermitente & Aminas, $\mathrm{Ca}, \mathrm{P}$ & Zona Urbana \\
\hline Jales Mineros & $\begin{array}{c}\text { Activa/ } \\
\text { potencial }\end{array}$ & Antropogénico & Difusa & Intermitente & $\mathrm{F}$ & Zona Urbana \\
\hline Descargas de aguas residuales & $\begin{array}{c}\text { Activa/ } \\
\text { potencial }\end{array}$ & Antropogénico & $\begin{array}{l}\text { Puntual/ } \\
\text { lineal }\end{array}$ & Intermitente & $\begin{array}{l}\text { SDT, } \mathrm{Cl}, \mathrm{N} \text {, } \\
\text { Comp. Org. }\end{array}$ & Zona Urbana \\
\hline Disposición de residuos sólidos & $\begin{array}{c}\text { Activa/ } \\
\text { potencial }\end{array}$ & Antropogénico & Puntual & Intermitente & Metales & $\begin{array}{l}\text { Zona Urbana } \\
\text { Zona Agrícola }\end{array}$ \\
\hline Formaciones Geológicas & $\begin{array}{c}\text { Activa/ } \\
\text { potencial }\end{array}$ & Natural & Difusa & Intermitente & $\mathrm{Cr}, \mathrm{F}, \mathrm{As}$ & $\begin{array}{l}\text { Zona Urbana } \\
\text { Zona Agrícola } \\
\text { Zona Industrial }\end{array}$ \\
\hline Gasolineras & Potencial & Antropogénico & Puntual & Intermitente & BTEX & $\begin{array}{l}\text { Zona Urbana } \\
\text { Zona Agrícola } \\
\text { Zona Industrial }\end{array}$ \\
\hline
\end{tabular}


$\mathrm{SO}_{4}^{-2}, \mathrm{Cl}, \mathrm{CE}, \mathrm{CT}$, GA que por su amplia distribución en esta zona se considera que estas potenciales fuentes de contaminación son de tipo difusa (Moran-Ramírez, 2010). Anomalías importantes en la zona urbana se localizan en norias, en el caso de los $\mathrm{NO}_{3}^{-1}$ y CT rebasa el límite permisible para consumo humano de la NOM-127SSA1-1994.

La principal área contaminada de estos parámetros se encuentra en las proximidades del panteón municipal de SGS; así como, en la zona centro de la ciudad (Figura 5).
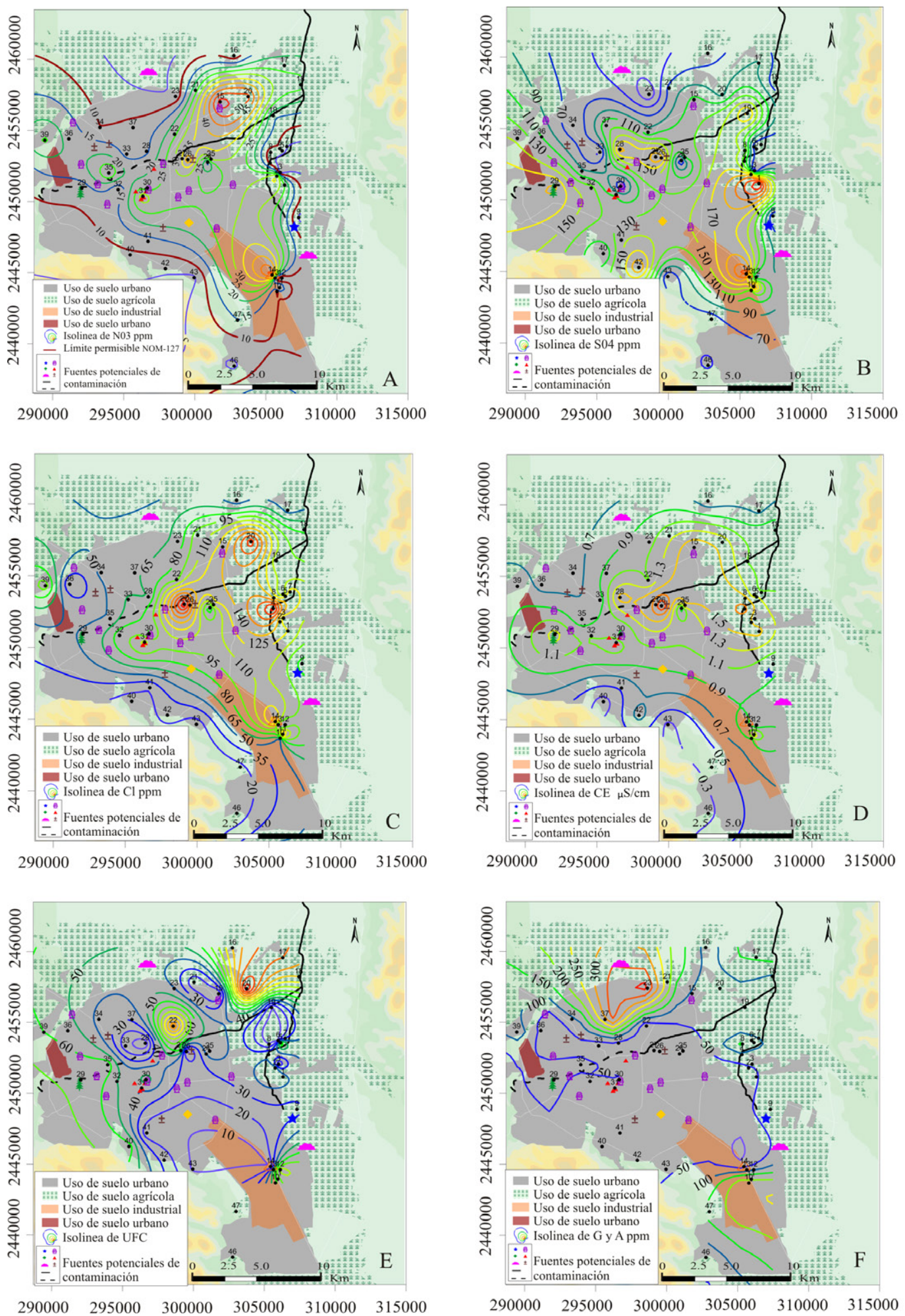

$290000 \quad 295000 \quad 300000 \quad 305000 \quad 310000 \quad 315000$ Figura 5. Distribución espacial de A: $\mathrm{NO}_{3}^{-1}, \mathrm{~B}: \mathrm{SO}_{4}^{-2}, \mathrm{C}: \mathrm{Cl}$, D: CE, E: CT y F: G y A, basados en Moran-Ramírez, 2010. 
El acuífero colgado en la zona urbana recibe una importante recarga inducida ocasionado por las fugas en las redes de agua potable y de drenaje (COTAS y CONAGUA, 2005), aportando cantidades significativas de estos contaminantes al acuífero.

Como se ve en la Figura 6, de manera puntual se identificaron fuentes de contaminación que generan $\mathrm{Hg}$, $\mathrm{Ba}, \mathrm{Sr}, \mathrm{Cd}, \mathrm{Pb}$ y Ag, siendo el primero de estos elementos el que sobrepasa el límite permisible para consumo humano de la NOM-127-SSA1-1994. En la Figura 7, también se detectaron valores importantes de $\mathrm{Rb}, \mathrm{Co}, \mathrm{Cu}, \mathrm{Fe}$ y $\mathrm{As}$, siendo este último el que sobrepasa el límite permisible para
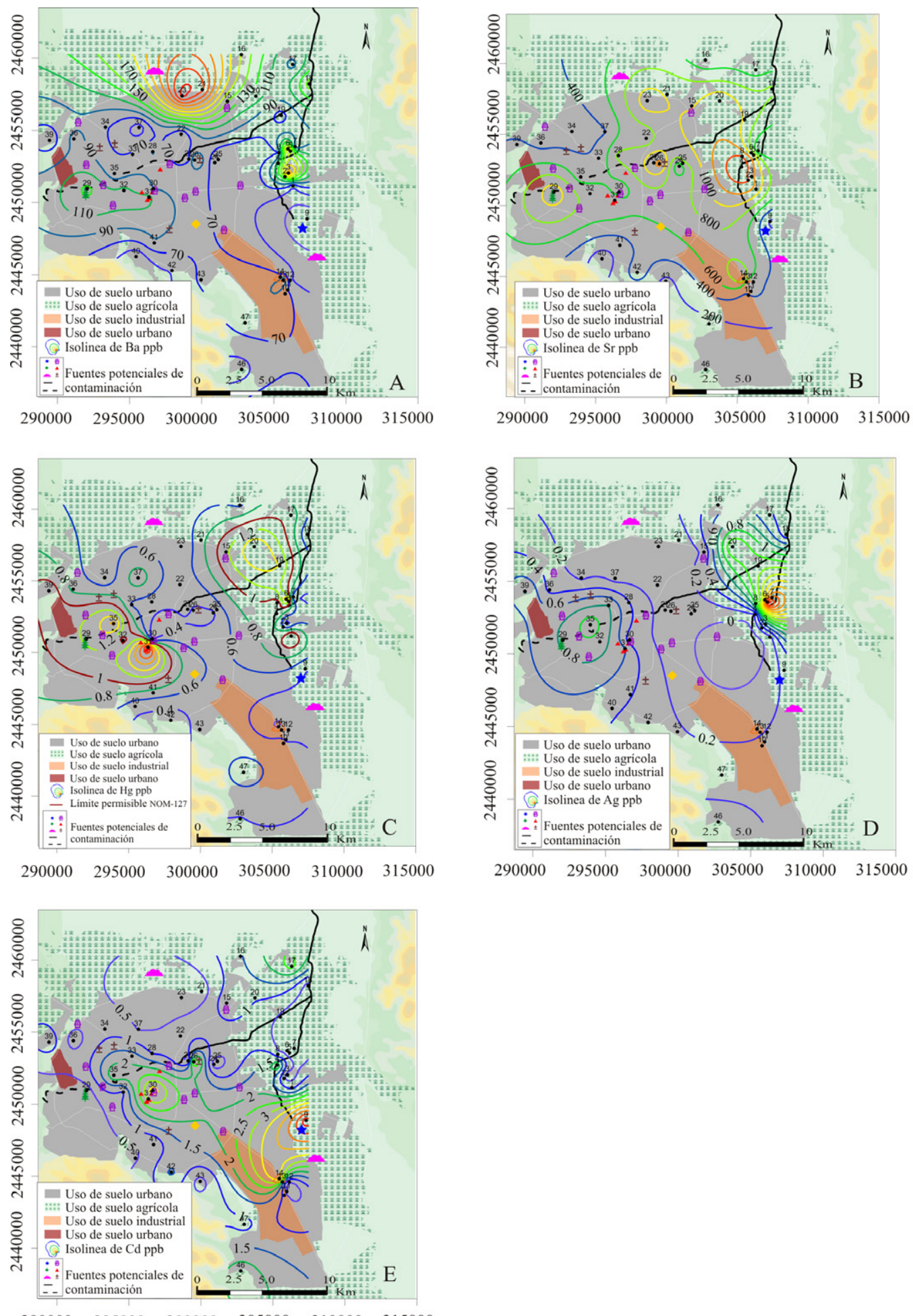

$290000 \quad 295000 \quad 300000 \quad 305000 \quad 310000 \quad 315000$

Figura 6. Distribución espacial de A: $\mathrm{Ba}^{+2}, \mathrm{~B}: \mathrm{Sr}^{+2}, \mathrm{C}: \mathrm{Hg}^{+2}, \mathrm{D}: \mathrm{Ag}^{+}, \mathrm{E}: \mathrm{Cd}^{+2}$, basados en Moran-Ramírez, 2010. 
consumo humano de la NOM-127-SSA1-1994.

Cabe mencionar que la presencia de metales pesados no es común en zonas urbanas; sin embargo, los niveles detectados en algunos sitios pueden estar relacionados a las actividades mineras realizadas a partir de la fundación de la ciudad en el siglo XVII (Keiser-Schlittler, 1992; Galván-
Arellano, 1999; IMPLAN, 2009).

Otra fuente de estos metales puede ser la presencia de talleres dentro de la zona urbana; así como la dispersión aérea de partículas. Lo cual explica la distribución de diferentes metales reportados en el presente trabajo. Cabe mencionar que el $\mathrm{Ba}$ y $\mathrm{Sr}$, también son utilizados en la
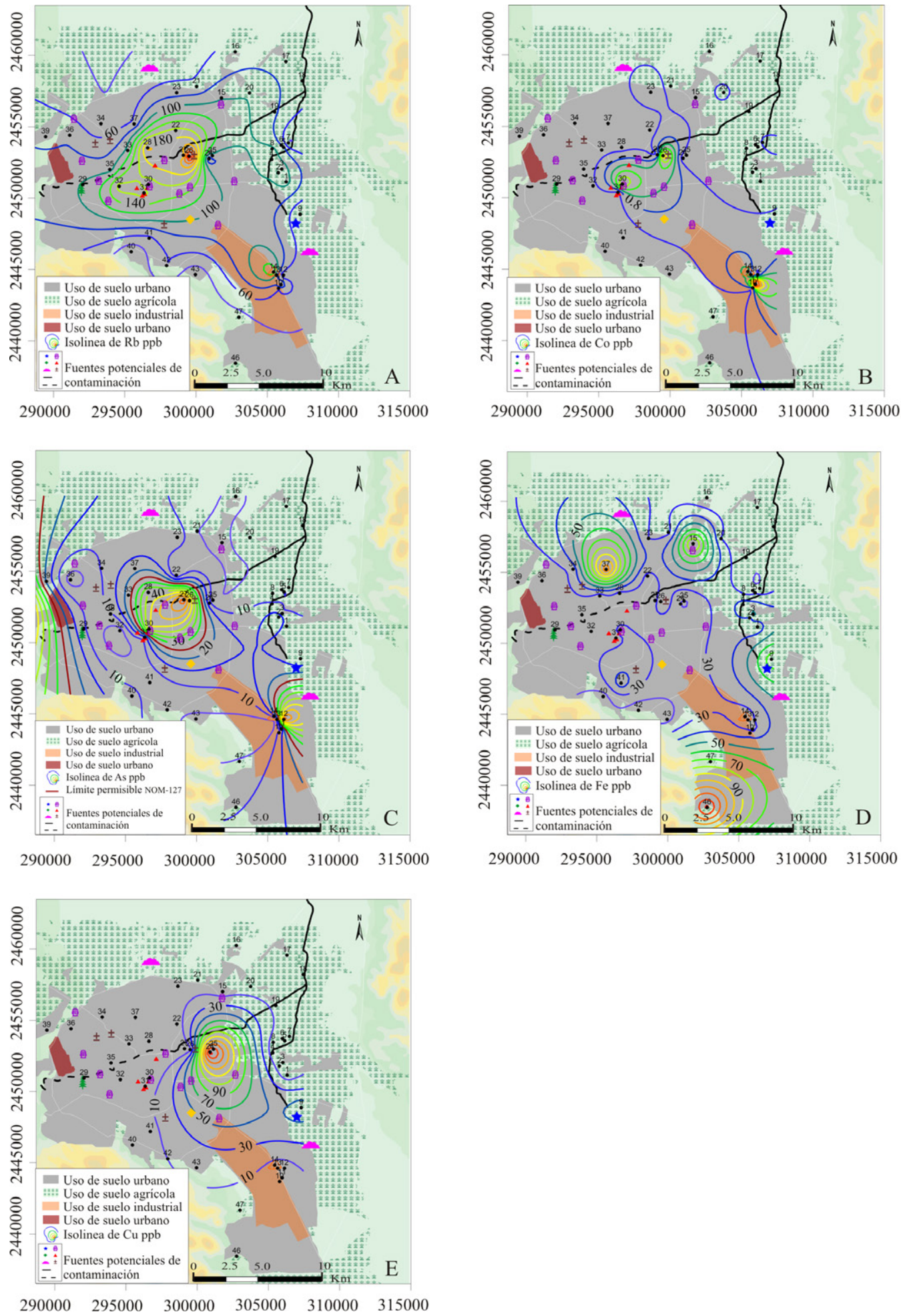

Figura 7. Distribución espacial de A: $\mathrm{Rb}^{+1}, \mathrm{~B}: \mathrm{Co}^{+2}, \mathrm{C}: \mathrm{As}^{+3} \mathrm{D}: \mathrm{Fe}^{+3}$, y E: $\mathrm{Cu}^{+2}$, basados en Moran-Ramírez, 2010. 
pirotecnia, actividad que ha sido reportada en la periferia de SLP (IMPLAN, 2009).

El Mn se detectó en el Parque Morales (Figura 8), el cual se encuentra cercano a una zona de uso de suelo industrial minero que actualmente emite partículas al ambiente durante sus procesos.

En el área de estudio, el comportamiento de las fuentes de contaminación es complejo. Generalmente pueden estar sujetos a procesos de atenuación natural (Gutiérrez-Ruíz et al., 2005); pueden ser transportados vía aérea (AragónPiña et al., 2006) y también pueden ser transportados por la acción fluvial.

Una vez que estos se depositan pueden ser afectados por el dinamismo del nivel freático del acuífero colgado, el cual es de poca profundidad, la carga contaminante puede ser transportada por el flujo subterráneo que va de SO a NE (COTAS y CONAGUA, 2005; IPICYT, 2010), de tal manera que pueden encontrarse anomalías en áreas diferentes a su sitio de origen.
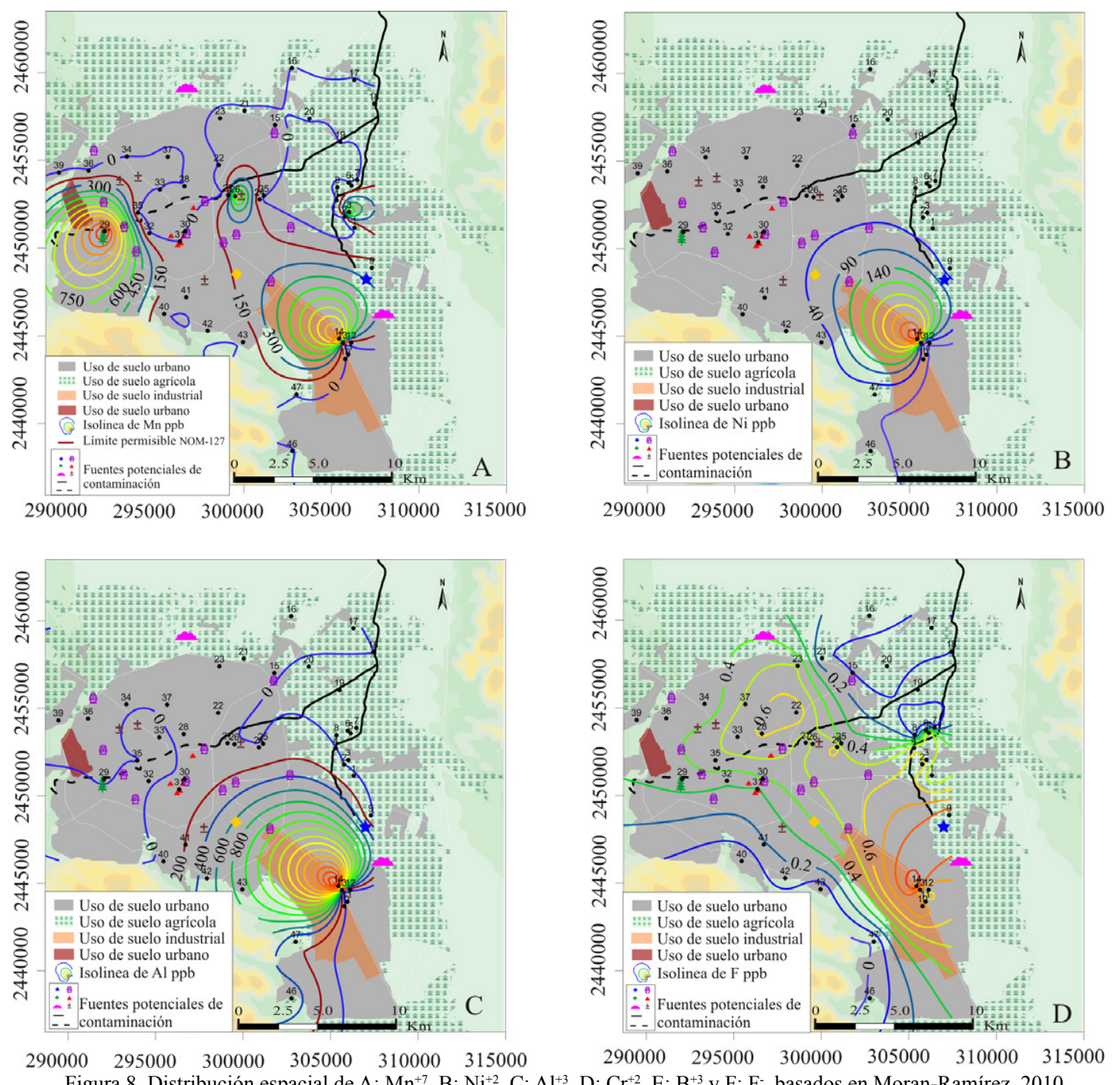

Figura 8. Distribución espacial de A: $\mathrm{Mn}^{+7}, \mathrm{~B}: \mathrm{Ni}^{+2}, \mathrm{C}: \mathrm{Al}^{+3}, \mathrm{D}: \mathrm{Cr}^{+2}, \mathrm{E}: \mathrm{B}^{+3}$ y F: F- basados en Moran-Ramírez, 2010.

\subsubsection{Contaminación en zonas agrícolas}

Las fuentes potenciales de contaminación presentes en la zona agrícola de SGS son panteones, canales con aguas residuales, disposición de residuos sólidos (Figura 1; Tabla 4), debido a que el retorno de riego es un factor importante en la movilidad de los contaminantes en este uso de suelo. $\mathrm{Su}$ contaminación característica es de tipo difusa en los $\mathrm{NO}_{3}^{-1}, \mathrm{SO}_{4}^{-2}$ y materia orgánica. Derivado de estas fuentes potenciales de contaminación se detectaron niveles importantes de $\mathrm{NO}_{3}{ }^{-1}, \mathrm{SO}_{4}^{-2}, \mathrm{Cl}^{-}$y $\mathrm{CE}$ (Figura 5). Los valores más altos se presentan en las proximidades de los canales abiertos.

Este tipo de contaminantes son derivados del uso de aguas residuales, fertilizantes y agroquímicos en la actividad agrícola.

Algunos metales como $\mathrm{Ba}, \mathrm{Sr}, \mathrm{Hg}, \mathrm{Ag}, \mathrm{Cd} \mathrm{Pb}, \mathrm{Zn}$ y Li se presentan de manera puntual en sitios cercanos a canales abiertos, derivados del antiguo Tanque Tenorio (Figuras 6 y 9), cuyas aguas provienen de la zona industrial (COTAS y CONAGUA, 2005).

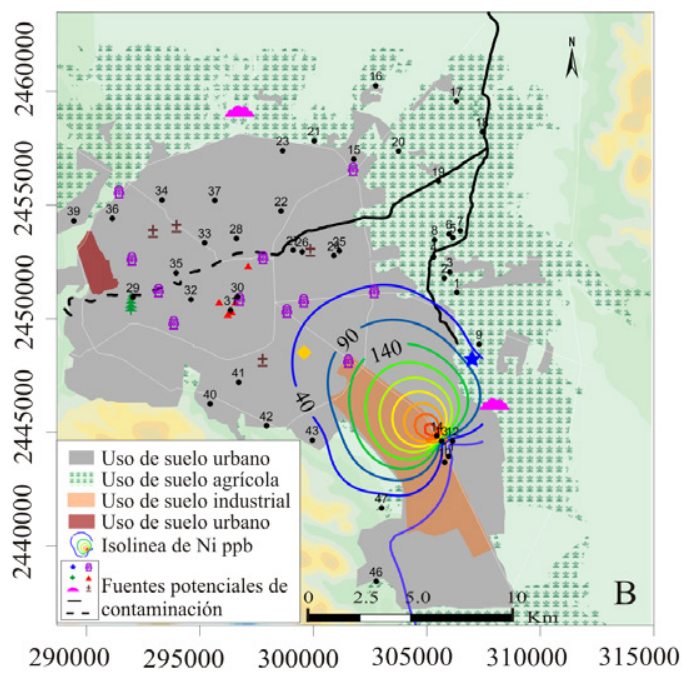


Origen de la calidad del agua del acuífero colgado y su relación con los cambios de uso de suelo

Tabla 4. Tipos de fuentes potencialmente contaminantes de acuerdo a su actividad, origen, geometría, temporalidad y tipo de soluto en la zona agrícola de San Luis Potosí- Soledad de Graciano Sánchez.

\begin{tabular}{|c|c|c|c|c|c|c|}
\hline Tipo de fuente & Actividad & Origen & Geometría & Temporalidad & Soluto & Uso de Suelo \\
\hline Terrenos agrícolas & Activa & Antropogénico & Difusa & Intermitente & Agroquímicos & Zona Agrícola \\
\hline Basureros activos & Potencial & Antropogénico & Puntual & Intermitente & Nitratos STD, Comp. Org. & Zona Agrícola \\
\hline Basureros clausurados & $\begin{array}{l}\text { Activa/ } \\
\text { potencial }\end{array}$ & Antropogénico & Puntual & Intermitente & Nitratos SDT, Comp. Org. & Zona Agrícola \\
\hline Ríos y canales de aguas residuales & Activa & Antropogénico & Lineal & Intermitente & $\mathrm{SDT}, \mathrm{Cl}, \mathrm{N}, \mathrm{Cr}$ & Zona Agrícola \\
\hline $\begin{array}{l}\text { Disposición de } \\
\text { residuos sólidos }\end{array}$ & $\begin{array}{l}\text { Activa/ } \\
\text { potencial }\end{array}$ & Antropogénico & Puntual & Intermitente & Metales & $\begin{array}{c}\text { Zona Urbana } \\
\text { Zona Agrícola }\end{array}$ \\
\hline Gasolineras & Potencial & Antropogénico & Puntual & Intermitente & BTEX & $\begin{array}{l}\text { Zona Urbana } \\
\text { Zona Agrícola } \\
\text { Zona Industrial }\end{array}$ \\
\hline
\end{tabular}
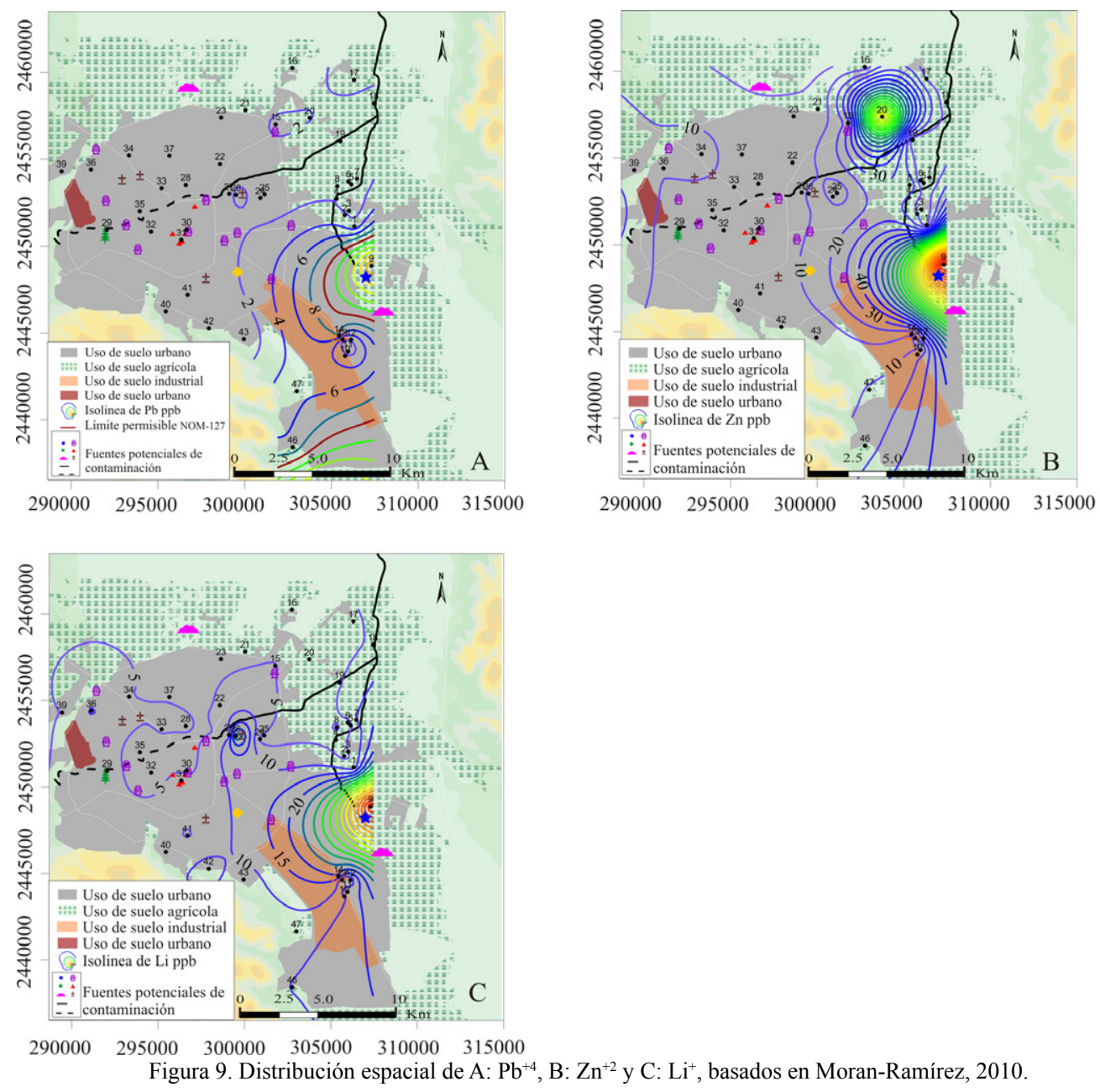

\subsubsection{Contaminación en zonas industriales}

Las fuentes potenciales de contaminación que existen en la zona industrial de San Luis Potosí están asociadas a disposición de lodos industriales, confinamiento de residuos industriales, terrenos industriales activos, terrenos industriales inactivos, gasolineras (Tabla 5). Derivado de estas fuentes potenciales de contaminación en la zona industrial se detectaron niveles importantes de $\mathrm{NO}_{3}^{-1}, \mathrm{SO}_{4}^{-2}$, $\mathrm{Cl}^{-}, \mathrm{CE}, \mathrm{CT}$ y $\mathrm{GA}$ (Figura 5).

Con una distribución puntual (Figuras 5 y 9) se detectó $\mathrm{Ba}, \mathrm{Sr}, \mathrm{Hg}, \mathrm{Ag}, \mathrm{Cd}, \mathrm{Co}^{+2}, \mathrm{Ni}, \mathrm{Al}$ y Fe. Es importante mencionar que el sitio ocupado por una bodega con uso industrial activo resulto anómalo en todos los parámetros analizados (Figuras 5 a 9). 
Tabla 5. Tipos de fuentes potencialmente contaminantes de acuerdo a su actividad, origen, geometría, temporalidad y tipo de soluto en la zona industrial de San Luis Potosí.

\begin{tabular}{|c|c|c|c|c|c|c|}
\hline Tipo de fuente & Actividad & Origen & Geometría & Temporalidad & Soluto & Uso de Suelo \\
\hline Disposición de lodos industriales & Potencial & Antropogénico & Puntual & Intermitente & $\begin{array}{l}\text { Comp. Orgánicos, metales, } \\
\text { sust. corrosivas }\end{array}$ & Zona Industrial \\
\hline Confinamiento de residuos industriales & Potencial & Antropogénico & Puntual & Intermitente & Metales & Zona Industrial \\
\hline Terrenos industriales activos & $\begin{array}{l}\text { Activa/ } \\
\text { potencial }\end{array}$ & Antropogénico & Puntual & $\begin{array}{c}\text { Pulso/ } \\
\text { intermitente }\end{array}$ & Metales, comp. orgánicos & Zona Industrial \\
\hline \multirow[t]{2}{*}{ Terrenos industriales inactivos } & Potencial & Antropogénico & $\begin{array}{c}\text { Puntual/ } \\
\text { Difusa }\end{array}$ & Intermitente & Metales, comp. orgánicos & Zona Industrial \\
\hline & & & & & & Zona Urbana \\
\hline \multirow[t]{2}{*}{ Gasolineras } & Potencial & Antropogénico & Puntual & Intermitente & BTEX & Zona Agrícola \\
\hline & & & & & & Zona Industrial \\
\hline
\end{tabular}

\section{Conclusiones}

La alteración al sistema hidrogeológico original desde el inicio de las actividades antropogénicas en el VSLP ha constituido un factor de alto impacto en la calidad actual del agua subterránea.

Los cambios hidrológicos al sistema natural en la región se habían dado de forma paulatina durante cuatro siglos a partir de la fundación de la ciudad; sin embargo, estos cambios han sido de forma drástica y en muy poco tiempo desde la segunda mitad del siglo veinte.

Así mismo, los cambios de uso de suelo en el valle desde los primeros asentamientos humanos hasta hoy en día, han propiciado un efecto de contaminación cruzada que vuelve más complejo el problema de contaminación en el agua subterránea.

Las fuentes potenciales de contaminación que existen en la zona industrial de San Luis Potosí están asociadas a disposición de lodos industriales, confinamiento de residuos industriales, terrenos industriales activos, terrenos industriales inactivos y gasolineras.

Las fugas de drenaje en la zona urbana alcanzan el nivel freático del acuífero colgado incrementando los valores de $\mathrm{NO}_{3}^{-1}, \mathrm{SO}_{4}^{-2}, \mathrm{Cl}^{-}, \mathrm{CE}, \mathrm{CT}$ y GA.

El origen de metales pesados ( $\mathrm{Hg}, \mathrm{Ag}, \mathrm{Ba}$ y $\mathrm{Sr})$ en el centro de la ciudad de San Luis Potosí, se debe a las actividades mineras en los patios de beneficio realizadas poco después de la fundación de la ciudad, que además se llevaron a cabo sin ningún tratamiento de manejo de los residuos.

El uso de agua residual y agroquímicos en las actividades agrícolas en la zona de Soledad de Graciano Sánchez impacta de manera importante al acuífero como lo demuestran los niveles de $\mathrm{NO}_{3}^{-1}, \mathrm{SO}_{4}^{-2}, \mathrm{Cl}^{-}, \mathrm{CE}$ y CT.

La presencia de metales ( $\mathrm{Hg}, \mathrm{Ag}, \mathrm{Ba}, \mathrm{Sr}, \mathrm{Cd}, \mathrm{Pb}, \mathrm{Zn}$ y Li) en la zona agrícola se asocia a sitios cercanos a canales abiertos derivados del antiguo Tanque Tenorio.

Otra fuente de aporte de metales pesados lo constituye el transporte aéreo de partículas de la zona industrial.

La industria activa, actualmente inmersa en la zona urbana, también contribuye con importantes valores de metales (Hg, Ag, Ba, Sr y Mn) al acuífero colgado, como lo demuestra su presencia en la zona oriente de la ciudad.

En la zona industrial se detectaron grandes anomalías en casi todos los metales pesados; las anomalías son de tipo puntual y corresponden a un terreno industrial activo.

Como se estableció, la contaminación en el acuífero colgado del VSLP es compleja, debido al origen de las diferentes fuentes, por lo que su solución resulta también compleja. Los datos presentados en este trabajo son un diagnóstico de la calidad del agua del acuífero colgado; sin embargo, se propone iniciar un monitoreo que permita establecer la hidrodinámica de los contaminantes antes tomar cualquier acción para mitigar sus efectos.

Algunas propuestas de acciones para aminorar los efectos de contaminación al acuífero son la instalación de plantas de tratamiento para los diferentes usos de agua, la modernización de la red de drenaje de la zona urbana, la instalación de rellenos sanitarios adecuados y la depuración primaria del agua residual utilizada para la agricultura. Cabe mencionar que los resultados esperados de estas acciones no serían inmediatos, sino a largo plazo, por lo que es necesario iniciarlas en el menor tiempo posible.

\section{Agradecimientos}

Queremos dar las gracias al proyecto 151666 , "Problemática socioambiental asociada al uso y manejo del agua en la cuenca del Valle de San Luis Potosí", el cual ha recibido financiamiento del Fondo de Ciencia Básica SEPCONACYT, en su edición 2010. También agradecemos al Programa de Agua y Sociedad del Colegio de San Luis, A. C. y a la División de Geociencias Aplicadas, del Instituto Potosino de Investigación Científica y Tecnológica, A. C., por el apoyo otorgado. 


\section{Referencias}

Aragón-Piña, A., Campos-Ramos, A., Leyva-Ramos, R., HernándezOrta, M., Miranda-Ortiz, N., Antoni Luszczewski-Kudra, A., 2006, Influencia de emisiones industriales en el polvo atmosférico de la ciudad de San Luis Potosí, México: Revista Internacional de Contaminación Ambiental, 22, 5-19.

Armienta-Hernández, M.A., Rodríguez-Castillo, R., Ceniceros N., Juárez F., Cruz O., 1996, Distribution, origin and fate soils in Guanajuato Mexico: Environmental Pollution, 9, 391-397.

Barber, C., Otto, C.J., Bate, L.E., 1996, Evaluation of the relationship between land-use changes and groundwater quality in a water supply catchment, using GIS Technology: The GWELUP Wellfield, Western Australia: Hydrogeology Journal, 4, 6-19.

Bateman, A., 1974, Yacimientos minerales de rendimiento económico: Barcelona, España, Ediciones Omega, 975 p.

Cardona-Benavides, A., 2007, Hidrogeoquímica de sistemas de flujo regional, intermedio y local resultado del marco geológico en la Mesa Central: reacciones, procesos y contaminación: México, D.F., México, Instituto de Geofísica, Universidad Nacional Autónoma de México, tesis doctoral, $252 \mathrm{p}$.

Cardona-Benavides, A., 1990, Caracterización físico-química y origen de los sólidos disueltos en el agua subterránea en el Valle de San Luis Potosí: su relación con el sistema de flujo: Monterrey, México, Universidad Autónoma de Nuevo León, tesis de maestría, 97 p.

Carrillo-Rivera J.J., Armienta, M.A., 1989, Diferenciación de la contaminación inorgánica en las aguas subterráneas del valle de la ciudad de San Luis Potosí, S.L.P., México: Geofísica Internacional, 28, 763-783.

Carrillo-Rivera, J.J., Clark, I.D., Fritz P., 1992, Investigating recharge of shallow and paleo-groundwaters in the Villa de Reyes basin, SLP, Mexico, with environmental isotopes: Applied Hydrogeology, 1, 35-48.

Carrillo-Rivera, J.J., Cardona-Benavides, A., Moss, D., 1996, Importance of the vertical component of groundwater flow: a hydrochemical approach in the valley of San Luis Potosí, México: Journal of Hydrology, 185, 23-44.

Carrillo-Rivera, J.J., Cardona-Benavides, A., Edmunds, W.M., 2002. Use of abstraction regime and knowledge to control high-fluoride concentration in abstracted groundwater: San Luis Potosí basin, Mexico: Journal of Hydrology, 261, 24-47.

Carrillo-Rivera, J.J., Cardona-Benavides, A., Huizar-Álvarez, R., GranielCastro, E., 2008, Response of the interaction between groundwater and other components of the environment in Mexico: Environmental Geology, 55, 303-319.

Charbonneau, R., Kondolf, G.M., 1993, Land use change in California, USA: Nonpoint source water quality impacts: Environmental Management, 17, 453-460.

Comité Técnico del Agua Subterránea (COTAS), Comisión Nacional del Agua (CONAGUA), 2005, Estudio técnico respecto a las condiciones geohidrológicas y sociales del acuífero 2411 "San Luis Potosí” en el Estado de San Luis Potosí: Comité Técnico de Agua Subterránea del Acuífero del Valle de San Luis Potosí, reporte, 74 p.

Cox, M.E., Hillier, J., Foster, L., Ellis, R., 1996, Effects of a rapidly urbanizing environment on groundwater, Brisbane, Queensland, Australia: Hydrogeology Journal, 4, 30-47.

De la Rosa-Charcas, A. Llanas-Rivera, M. A., 2000, Los grandes retos del Valle de San Luis: Secretaria de estudios y programas del comité ejecutivo estatal del Partido Revolucionario Demócrata: Secretaría de Estudios y Programas del CEE del PRD, Keiser Ediciones, San Luis Potosí, México, 136 p.

Dummer, M., Straaten, V., 1988, The influence of industrialization and hydrogeology on the quality of ground water and surface water in Bielefeld (case studies): Symposium on hydrological processes and water management in urban areas, 1988, Duisberg, FRG, 423-428.

Fetter, C.W., 1999, Applied Hydrogeology:Estados Unidos de América, Prentice Hall $4 \mathrm{t}^{\mathrm{h}}$ edition, $589 \mathrm{p}$.

Galván-Arellano, A., 1999, Arquitectura y urbanismo de la ciudad de San Luis Potosí en el siglo XVII: Facultad de Hábitat, Universidad
Autónoma de San Luis Potosí, San Luis Potosí, México, 268 p.

Gutiérrez-Ruíz, M., Mario Villalobos, Romero, F., Fernández-Lomelín, P., 2005, Natural attenuation of arsenic in semiarid soils contaminated by oxidized Arsenic wastes, en O’Day, P.A., Vlassopoulos, D., Meng, X., Liane G., Benning, L.G., (eds.), Advances in Arsenic Research: Washington D.C., Integration of Experimental and Observational Studies and Implications for Mitigation, American Chemical Society ACS Symposium Series, 915, 235-251.

Instituto Municipal de Planeación (IMPLAN), 2009, Plan Parcial de Conservación del Centro Histórico de San Luis Potosí 2009-2012. San Luis Potosí, S.L.P., Proyectos Municipales, H. Ayuntamiento de San Luis Potosí, 221 p.

Instituto Nacional de Geografía Estadística e Informática (INEGI), 2010, Conteo de Población y Vivienda 2010: México, Instituto Nacional de Geografía, Estadística e Informática, disponible en http://www3. inegi.org. $\mathrm{mx} /$ sistemas/mexicocifras/default.aspx src $=487 \& \mathrm{e}=24$, consultado 27 de Junio de 2011.

Instituto Potosino de Investigación Científica y Tecnológica A.C. (IPICYT), 2007, Estudio hidrogeológico de la porción oriental del Valle de San Luis Potosí: San Luis Potosí, S.L.P., IPICYT, reporte técnico elaborado para el Gobierno del Estado de San Luis Potosí, 174 p.

Instituto Potosino de Investigación Científica y Tecnológica A.C. (IPICYT), 2010, Diagnóstico del potencial del acuífero somero del Valle de San Luis Potosí como una fuente alternativa de abastecimiento: San Luis Potosí, S.L.P., IPICYT, reporte técnico elaborado para el Comité Técnico del Agua Subterránea (COTAS), 95 p.

Keiser-Shlittler, A., 1992. Breve Historia de la ciudad de San Luis Potosí: Keiser Editores, 230 p.

Liu, W.H., Zhau, J.Z., Ouyang, Z.Y., Soderlund, L., Liu, G.H., 2005, Impacts of sewage irrigation on heavy metal distribution and contamination in Beijing, China: Environmental International, 31, 805-812.

Medellín-Milán, P., 2003, Tanque Tenorio, diez años después: San Luis Potosí, México, Pulso, Diario de San Luis, Sección Ideas, publicado el 22 de Mayo de 2003, 4p.

Moran-Ramírez, J., 2010, Evaluación de la calidad química del agua en el acuífero somero de San Luis Potosí: San Luis Potosí, S.L.P., Universidad del Noreste, Área de Ciencias de Químico Biológicas, tesis de licenciatura, $68 \mathrm{p}$.

Naik, P.K., Tambe, J.A., Dehury, B.N., Tiwari, A.N., 2008, Impact of urbanization on the groundwater regime in a fast growing city in central India: Environmental Monitoring and Assessment, 146, 339-373.

Noyola-Medrano, M.C., Ramos-Leal, J.A., Domínguez-Mariani, E., Pineda-Martínez L.F., López-Loera, H., Carbajal, N., 2009, Factores que dan origen al minado de acuíferos en ambientes áridos: caso Valle de San Luis Potosí: Revista Mexicana de Ciencias Geológicas, 24, 395-410.

Peña, F., 2006, Abasto de agua a la ciudad de San Luis Potosí, en Barkin, D. (ed.), La Gestión del Agua Urbana en México: Guadalajara, México, Universidad de Guadalajara, 249-264.

Ramos-Leal, J.A., López-Loera, H., Martínez-Ruiz, V.J., Aranda-Gómez, J.J., 2007, Sucesión de eventos y geometría de la parte central del acuífero del graben de Villa de Reyes (San Luis Potosí, México) inferida a partir de datos geoeléctricos: Revista Mexicana de Ciencias Geológicas, 24, 31-46.

Ramos-Leal, J.A., 2002, Validación de mapas de vulnerabilidad acuífera e impacto ambiental: caso Río Turbio, Guanajuato: México, D.F., México, Instituto de Geofísica, Universidad Nacional Autónoma de México, tesis de doctorado, $106 \mathrm{p}$.

Robles, J., Armienta, M.A., 2000, Natural Chromium Contamination of Groundwater at Leon Valley, Mexico: Journal of Geochemical Exploration, 68, 167-181.

Schmidt, K., Sherman, I., 1987, Effect of Irrigation on Groundwater Quality in California: Journal of Irrigation and Drainage Engineering, 1, 113.

Secretaria de Medio Ambiente y Recursos Naturales (SEMARNAT), 2005, Informe sobre la situación del medio ambiente en México 2005, disponible en http://app1.semarnat.gob.mx/dgeia/informe_04/07 agua/recuadros/c_rec2_07.htm, consultado el 2 de Mayo de 2011. 
Secretaria de Medio Ambiente y Recursos Naturales (SEMARNAT), 2008 , Boletín del Archivo Histórico del Agua, 13, 40, 89 p.

Sheridan-Prieto, C., 2001, "Indios madrineros" - colonizadores tlaxcaltecas en el noroeste novohispano: Estudios de Historia Novohispana, 24, 15-51 p.

Tong, S.T.Y., Chen, W., 2002. Modeling the relationship between land use and surface water quality: Journal of Environmental Management, 66, 377-393.

United Nations Educational Scientific and Cultural Organization (UNESCO), 2004, Groundwater contamination inventory: a methodological guide, Project 3.1 (IHP-V): Paris, France, UNESCO, $162 \mathrm{p}$.
Wilson A.R., 1991, Environmental Risk: Identification and Management: Chelsea, Missouri, U.S.A., Lewis Publishers, 413 p.

Manuscrito recibido: Junio 3, 2011.

Manuscrito corregido recibido: Junio 15, 2012.

Manuscrito aceptado: Junio 28, 2012. 